\title{
DebrisLV Hypervelocity Impact Post-Shot Physical Results Summary
}

February 27, 2015

Patti M. Sheaffer ${ }^{1}$, Paul M. Adams ${ }^{2}$, Naoki Hemmi ${ }^{3}$, Christopher Hartney ${ }^{1}$

${ }^{1}$ Space Science Applications Laboratory

Physical Sciences Laboratories

${ }^{2}$ Space Materials Laboratory

Physical Sciences Laboratories

${ }^{3}$ Structural Mechanics Subdivision

Vehicle Systems Division

\section{Prepared for:}

Space and Missile Systems Center

Air Force Space Command

483 N. Aviation Blvd.

El Segundo, CA 90245-2808

Contract No. FA8802-14-C-0001

Authorized by: Space Systems Group 


\section{Report Documentation Page}

Public reporting burden for the collection of information is estimated to average 1 hour per response, including the time for reviewing instructions, searching existing data sources, gathering and maintaining the data needed, and completing and reviewing the collection of information. Send comments regarding this burden estimate or any other aspect of this collection of information,

including suggestions for reducing this burden, to Washington Headquarters Services, Directorate for Information Operations and Reports, 1215 Jefferson Davis Highway, Suite 1204, Arlington

VA 22202-4302. Respondents should be aware that notwithstanding any other provision of law, no person shall be subject to a penalty for failing to comply with a collection of information if it

does not display a currently valid OMB control number.

\begin{tabular}{l|l|l} 
1. REPORT DATE & 2. REPORT TYPE & 3. DATES COVERED \\
27 FEB 2015 & Final & -
\end{tabular}

27 FEB 2015

Final

4. TITLE AND SUBTITLE

DebrisLV Hypervelocity Impact Post-Shot Physical Results Summary

5a. CONTRACT NUMBER

DebrisLV Hypervelocity Impact Post-Shot Physical Results Summary

6. $\operatorname{AUTHOR}(\mathrm{S})$

Patti M. Sheaffer, Paul M. Adams, Naoki Hemmi, Christopher Hartney

7. PERFORMING ORGANIZATION NAME(S) AND ADDRESS(ES)

The Aerospace Corporation 2310 E. El Segundo Blvd. El Segundo, CA

90245-4609

9. SPONSORING/MONITORING AGENCY NAME(S) AND ADDRESS(ES)

Space and Missile Systems Center Air Force Space Command 483 N.

Aviation Blvd. El Segundo, CA 90245-2808

FA8802-14-C-0001

5b. GRANT NUMBER

5c. PROGRAM ELEMENT NUMBER

5d. PROJECT NUMBER

5e. TASK NUMBER

5f. WORK UNIT NUMBER

8. PERFORMING ORGANIZATION REPORT NUMBER

TOR-2014-03577

10. SPONSOR/MONITOR'S ACRONYM(S)

SMC

11. SPONSOR/MONITOR'S REPORT $\operatorname{NUMBER}(\mathrm{S})$

12. DISTRIBUTION/AVAILABILITY STATEMENT

Approved for public release, distribution unlimited

13. SUPPLEMENTARY NOTES

The original document contains color images.

14. ABSTRACT

15. SUBJECT TERMS

16. SECURITY CLASSIFICATION OF:

a. REPORT

unclassified

\author{
b. ABSTRACT \\ unclassified
}

\author{
17. LIMITATION \\ OF ABSTRACT \\ UU
}

unclassified

\begin{tabular}{l|l}
$\begin{array}{c}\text { 18. NUMBER } \\
\text { OF PAGES } \\
\mathbf{5 4}\end{array}$ & 19a. NAME OF \\
& RESPONSIBLE PERSON \\
&
\end{tabular}




\section{Foreword}

The DebriSat experiment was a unique opportunity for several segments of the Space Debris community to participate jointly in an effort designed to answer fundamental questions about the assumptions contained in current-generation orbital-impact breakup models, questions which have emerged in the last several years and are of importance to the future of space science. All work proceeded by the joint effort of all participants toward the common goal of observing and interpreting new, relevant phenomena in each participants' field of expertise. The observed phenomena will enhance both understanding and accuracy of future hypervelocity impact breakup models. 


\section{Acknowledgments}

The data for this document could not have been acquired without the active help and support of NASA (J.-C. Liou, Robert Markowicz); Jacobs Technologies (John Opiela, Heather Cowardin); and The Aerospace Corporation's Physical Sciences Laboratories, Chief Engineer, and Orbital Mechanics personnel (Paul Adams, Gouri Radhakrishnan, Marlon Sorge, Charles Griffice, Mary Ellen Vojtek), and others. The technical and experiential inputs of Mr. Brian Roebuck and Mr.

David Woods Arnold Engineering Development Complex (AEDC) played a key role in designing DebrisLV and interpreting the results. The very difficult task of carefully removing and marking the debris shown herein is gratefully acknowledged to be the hard work of Professor Norman Fitz-Coy and his students of the University of Florida, as well as Dr. J.-C. Loiu, Marlon Sorge, and David Woods of AEDC. 


\begin{abstract}
The DebrisLV ("Launch Vehicle") target was a value-added, companion target to the full-scale satellite target (i.e., DebriSat) in the joint NASA/SMC-funded DebriSat Hypervelocity Impact Experiment. DebrisLV was conceived and built at The Aerospace Corporation in the Remote Sensing Department of the Space Sciences Applications Laboratory, Laboratory Operations, Engineering and Technology Group. DebrisLV was designed to yield new information on the physics and mechanics of hypervelocity breakup. DebrisLV was designed in close collaboration with NASA and the Hypervelocity Impact expert-experimentalists at the Arnold Engineering Development Complex (AEDC) at Arnold AFB in order to represent the full-scale hypervelocity impact behavior of the current population of derelict launch vehicle upper stages in orbit. Although substantial forensic analysis remains to be done, the DebrisLV test has been extraordinarily fruitful in yielding new understanding of hypervelocity impacts.
\end{abstract}




\section{DebrisLV Hypervelocity Impact Post- Shot Physical Results Summary}

February 11, 2015

Patti M. Sheaffer ${ }^{1}$, Paul M. Adams ${ }^{2}$, Naoki Hemmi ${ }^{3}$, Christopher Hartney ${ }^{1}$

${ }^{1}$ Space Science Applications Laboratory

Physical Sciences Laboratories

2Space Materials Laboratory

Physical Sciences Laboratories

${ }^{3}$ Structural Mechanics Subdivision

Vehicle Systems Division 


\section{EXECUTIVE SUMMARY}

1. Full-scale hypervelocity test tests, such as DebriSat and DebrisLV, are required in order to avoid dataartifacts caused by the intrinsic nonlinear size-scaling of some materials properties (e.g., fracture toughness) and impact physics (e.g., energy transfer). DebrisLV used full-thickness materials, standard rivets, and a flight-qualified PAM-D upper stage Nutation Control Thruster (NCT) assembly. A low-density, heavy-walled projectile realistically simulated a large fragment or miniature vehicle to obtain realistic energy transfer into the DebrisLV target.

2. The impact energy/mass ratio was $>800 \mathrm{~J} / \mathrm{g}, 22 x$ the standard criterion for "catastrophic collision" as defined by six baseline IADC members [1]. We seek to understand the physics of impacts in this energy range and how it gives rise to various fragment size distributions. (Another recent experiment which is not discussed herein has probed the $250 \mathrm{~J} / \mathrm{g}$ energy range.)

3. Despite very high energies, no unusual metal mechanical-fragmentation mechanisms were observed in Debris $L V$; ductile failure was the dominant mechanism for this exclusively metallic structure. No mechanism for producing small particles by direct mechanical fragmentation is observed in the debris which has been examined to date. The soft-catch foam has not yet been processed to extract fragments; however, three unexpected mechanisms for yielding small fragments were revealed in two different analyses (see below).[2-4]

4. It is estimated that on the order of $100 \mathrm{~g}$ of metallic aluminum was vaporized and at least partially converted to hypervelocity plasma by the impact (i.e., plasma jets). This explains the need for assumed energy sinks in current fragmentation models [8]. Equally importantly, metal vapor/plasma plays a previously unappreciated, key role in full-scale impacts: (1) the plasma blast velocity was estimated at 2-4 times the incoming projectile speed and appeared to be an important mechanism for internal forcetransmission and fragmentation of stout metallic structures; (2) microanalysis studies [5,6] reveal that metal vapor can give rise to very large numbers of micro- and nano-particles via condensation and/or homogeneous nucleation; (3) liquid-aluminum, formed by condensation from the vapor onto 


\section{EXECUTIVE SUMMARY (continued)}

solid metal surfaces, yields thin (tens to hundreds of $\mu \mathrm{m}$ thickness) metal flakes, up to a centimeter or more in size. These flakes are only weakly bound to the metal substrate and therefore tend to detach due to differential thermal stress, releasing centimeter-scale high-energy orbiting fragments. (Formation of these flakes was also observed in a recent lower-energy, full scale collision.)

5. The importance of plasma jetting: We observed clear evidence for the formation of plasma/vapor jets powerful enough to easily pierce and fragment nearby aluminum and titanium pressure vessels. Although the existence of elemental plasma/vapor has been spectroscopically demonstrated by many authors in hypervelocity impacts, to our knowledge, this provides the first clear evidence of the importance of plasma jetting as a primary fragmentation mechanism. This mechanism is probably only clearly observable in full-scale hypervelocity impacts. Condensed-phase jet formation is reasonably well-predicted by hydrocode models, although the vapor phase aspect is yet to be included. [7, 9-11]

6. The importance of the Primary Fragment Cone (PFC): The PFC, typically observed in full-scale hypervelocity impacts (i.e., at AEDC) provides a useful "rule-of-thumb" guide to understanding the expected fragmentation of a real-world structure. It can also provide insight into particle size distribution yields in conjunction with post-impact assessment activities relying on current statistical models (e.g., Ref. 2, 8). In the case of the DebrisLV test, following impact, the outbound cone of primary fragments carried a great deal of energy and momentum and easily destroyed stout metal structures within the cone (plus and minus $\sim 35^{\circ}$, centered in the impact vector), while leaving structures outside the cone relatively unfragmented. It is also noted that the PFC appears to be wellmodeled by current-generation hydrocodes, suggesting the forensic utility of such code analyses of cone generation; however, current-generation hydrocodes do not directly model the important plasma-jetting modes (see \#4 and \#5, above).

6. More sophisticated corrobration of the observed DebrisLV results with hydrocode results will yield better forensics. 


\section{PURPOSE AND NEED}

- Present latest physical results of DebrisLV hypervelocity impact experiment, including important new full-scale results, which appear to be unique within the hypervelocity breakup community

- Relate these results to current and future SMC needs regarding impact fragmentation assessments and orbital debris modeling for space situational awareness and rapid predictions in the event of future orbital collisions

- Describe future work required to leverage the new knowledge gained from the DebrisLV experiment, including participation in expected full-scale test Targets of Opportunity (TOOs) at AEDC 


\section{TECHNICAL RESULT GOALS/VALUE ADDED}

- Refine physics and mechanics knowledge of impact fragmentation mechanisms and results

- Learn what Hydrocode models already do relatively well (i.e., condensed-phase modeling) and leverage these capabilities to better understand the observed physical break-up mechanisms

$>$ Insights into the physical breakup mechanisms will help improve breakup models.

- Potentially support JSPOC with estimates of unobservable debris, which result from the new mechanisms reported herein, during assessment activities following an on-orbit collision.

- Increased prediction accuracy for untrackable debris; improved understanding of the detailed mechanisms of momentum transfer and fragmentation in orbital impacts. 


\section{PROGRAM BACKGROUND}

- DebriSat is an ongoing joint NASA/SMC/Aerospace experiment to gain needed insights into hypervelocity impact breakups of satellites utilizing modern design principles

- Designed and built at University of Florida

- DebrisLV was recognized as value-added by NASA/SMC to understand impacts involving the $150+$ upper stages currently disposed in low Earth orbit

- Designed and built at The Aerospace Corporation

- Incorporated a flight-grade hydrazine thruster (a PAM-D Nutation Control Thruster, or NCT assembly)

- DebrisLV and DebriSat experiments were carried out consecutively at Arnold Engineering Development Complex at Arnold AFB, TN, on April 1 and April 15, 2014.

- Both experiments utilized polyurethane "soft-catch" foam to save fragments and fragment distributions/trajectories, but tended to mask microanalysis results

- DebriSat and DebrisLV Foam and DebriSat post-shot fragments shipped to University of Florida

- DebrisLV post-shot free fragments sent to The Aerospace Corporation

- This document focuses primarily on the DebrisLV results 


\section{CORE TECHNICAL MOTIVATION FOR DEBRISLV}

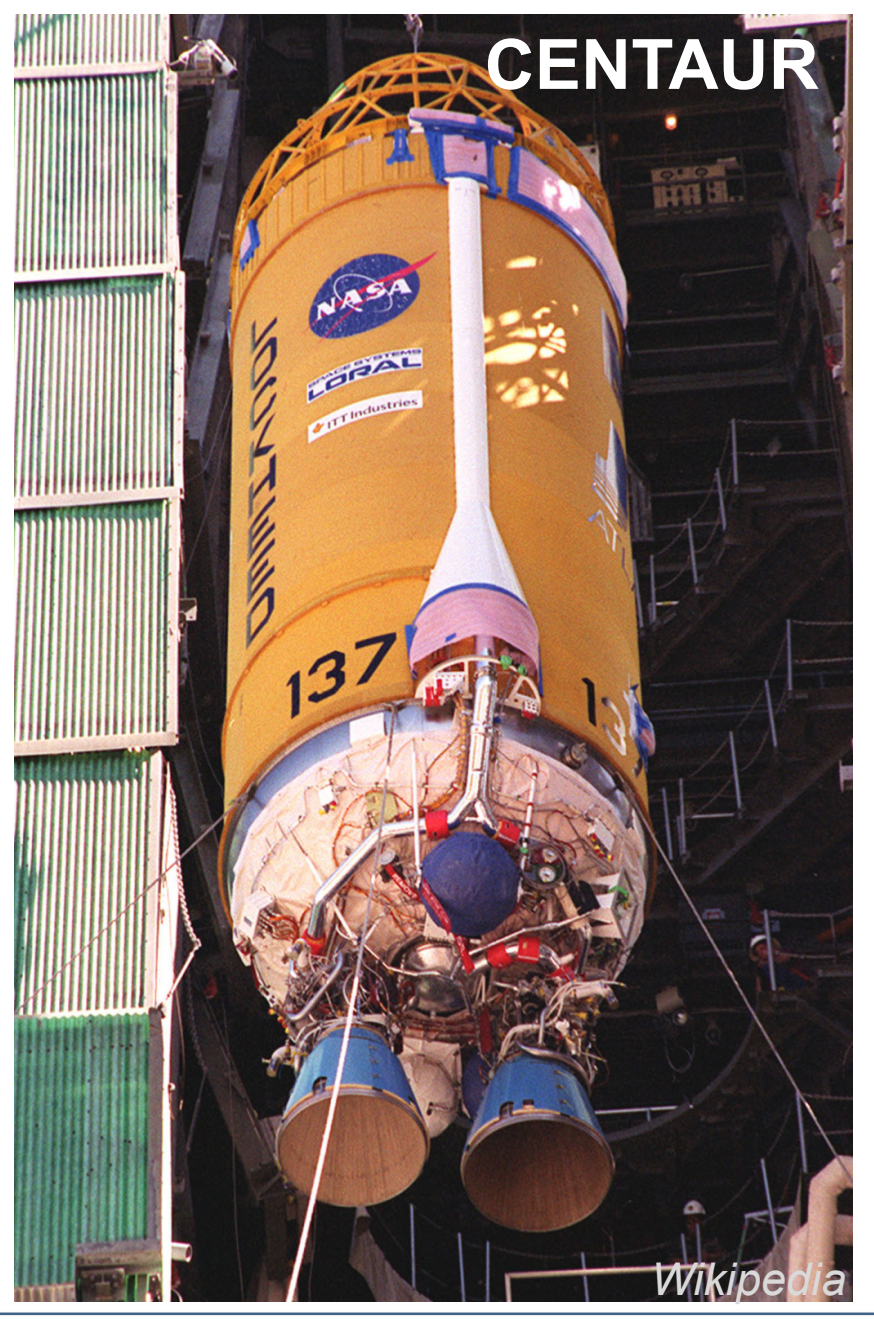

Nozzles, Throats, Combustion Chambers, Turbopumps, LOX/Fuel Lines, Engine Compartment Fittings, Tank Baffles, Pressurization Tanks, Viaducts, Stage Couplers, Valves, Fittings, etc.

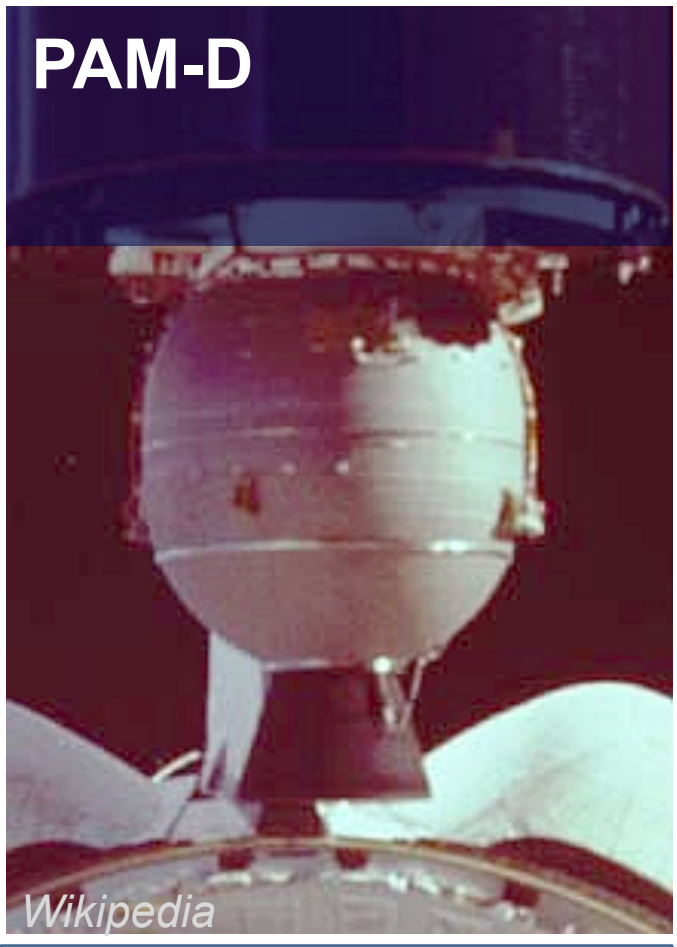

Nozzle, Throat, Tank, Strapon Motors
- “Tank-like" upper stages which fragment very differently under identical orbital impacts due to construction and materials differences.

- The physical mechanisms giving rise to the fragmentation differences are beginning to be understood from forensic analyses of DebrisLV.

- DebrisLV observations may help to support future post-event assessment activities, as well as contribute to upgrades of orbital fragmentation models. $[2,8]$

- Combined forensics, computer models, and microanalysis studies provide important insights. 


\section{INTRODUCTION}

- DebrisLV (LV = "Launch Vehicle") was designed to be both a realistic simulation of the $>\mathbf{1 5 0}$ orbiting upper stages, and a simple enough structure to support detailed forensic and physical post-analysis.

- "Dry Run" for experimental execution and collection processes for DebriSat

- Provide greater insight into the distinctive characteristics of upper-stage fragmentations in on-orbit events

- Detect and describe poorly understood mechanisms of hypervelocity fragmentation at sufficient level of detail to support the enhancement of existing orbital fragmentation models

- Several important results are presented

- Details of physical mechanisms which transfer impact forces within structures

- Details of physical mechanisms for generation of untrackable fragments

- Observations of a potential role of Strength-of-Materials in fragmentation

- Observations on fastener-release from upper stages (e.g., rivets vs. screws and metals vs. composites) 


\section{INTRODUCTION (continued)}

- DebrisLV experiment activities:

- Ongoing literature survey for major types of knowledge about orbital and hypervelocity impact experiments

- Propose anticipated and potential phenomenological results; design reasonable experiments to record evidence for, or against, expected results

- Repeatedly interact with AEDC Hypervelocity Impact expert experimentalists (B. Roebuck, D. Woods) on "previous and typical" AEDC results and their applicability to DebrisLV

- Consult with Aerospace experts for plasma physics, microanalysis, materials science, and forensics

- Develop methods to calibrate results of emissions from plasmas generated-develop experimental suite of instruments

- Modify and improve DebrisLV experimental design in real time

- Help meet soft-catch foam installation deadlines on-site

- Recover important post-experiment data (large, free fragments of DebrisLV)

- Develop analytical tools to interpret post-experimental data in the context of SMC needs for enhanced orbital debris generation models and predictions/assessments

- Synthesize and integrate data from video, microanalysis, and forensics into scientific by explanations of observed novel phenomena 


\section{INTRODUCTION (continued)}

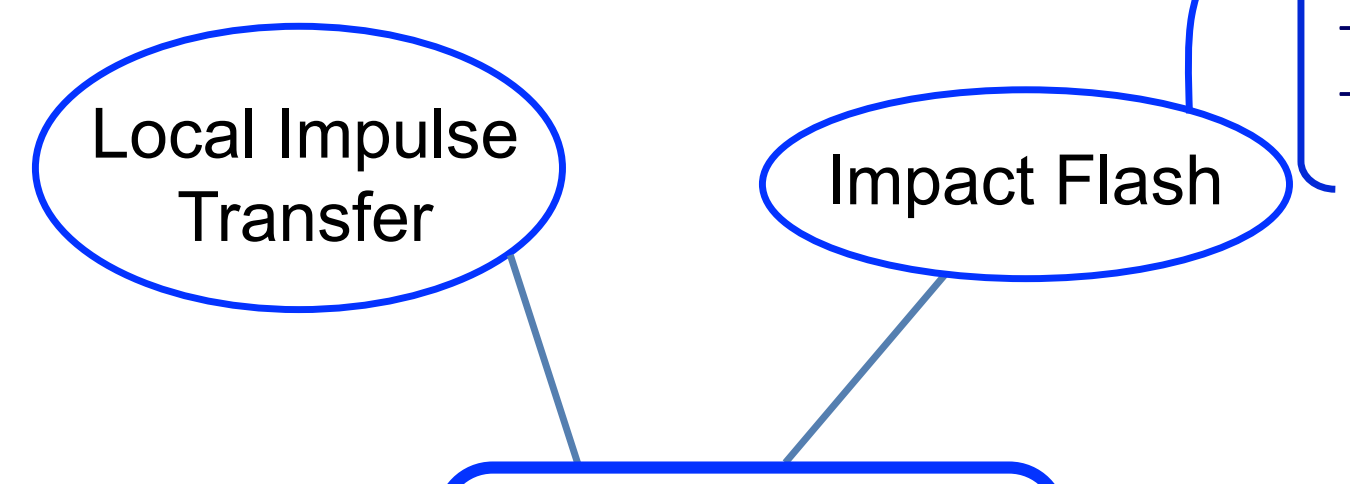

- Difference between DebriSat and DebrisLV

- Determine actual impact parameters

- Understand Probability of Outcome

- Mixed phase flow, rapid cooling, fragmentation, (melting, ionization, vaporization, etc.)

Structural

Energy Transfer)

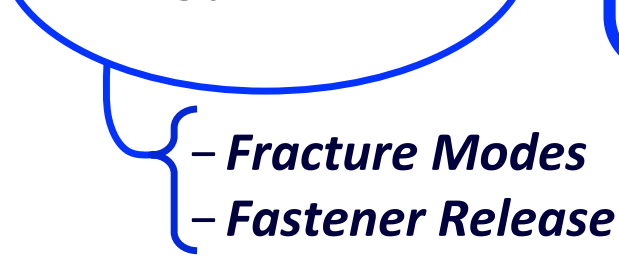

Aluminum Vapor/ Plasma Blast (upper stages)

\section{BLUE: Observations}

\section{Reported Here}

The observation of metal vapor is not new, but this document shows its importance to fragment-producing processes which have

not been appreciated in the past.

\section{Nano-spheres}

- Metal Flakes (small)

- Metal Spheres (small)

- Metal gases (?)

- Mixed phase flow, rapid cooling, fragmentation, melting,

ionization, vaporization, etc. [12]
Standard

Fragmentation

Impact Fragment Counting and Estimation

Analyses 


\section{BRIEF SURVEY/EXAMPLE DATA RESULTS}

$>$ This is a quick survey intended only to provide context; more detailed technical briefings are available by other investigators/SMEs

- Hydrocode Examples (particles and temperatures)

- DebrisLV Overall Geometry and Construction

- DebrisLV Main Tank Fragmentation (cratering)

- DebrisLV Strapon Vapor/Plasma Blast Evidence

○ Micro- and Nano-particles, Vapor Mixing

○ Energetics and Layout 


\section{Hydrocode and Primary Fragment Cone (PFC)}

Hydrodynamic Modeling for Hypervelocity Impact Fragmentation

- Hydrocode : CTH (Sandia Hydrodynamic code)

- Material Equations: Johnson-Cook Fracture Model and Grady-Kipp Dynamic Fragmentation Model (no vapor/plasma models are available)
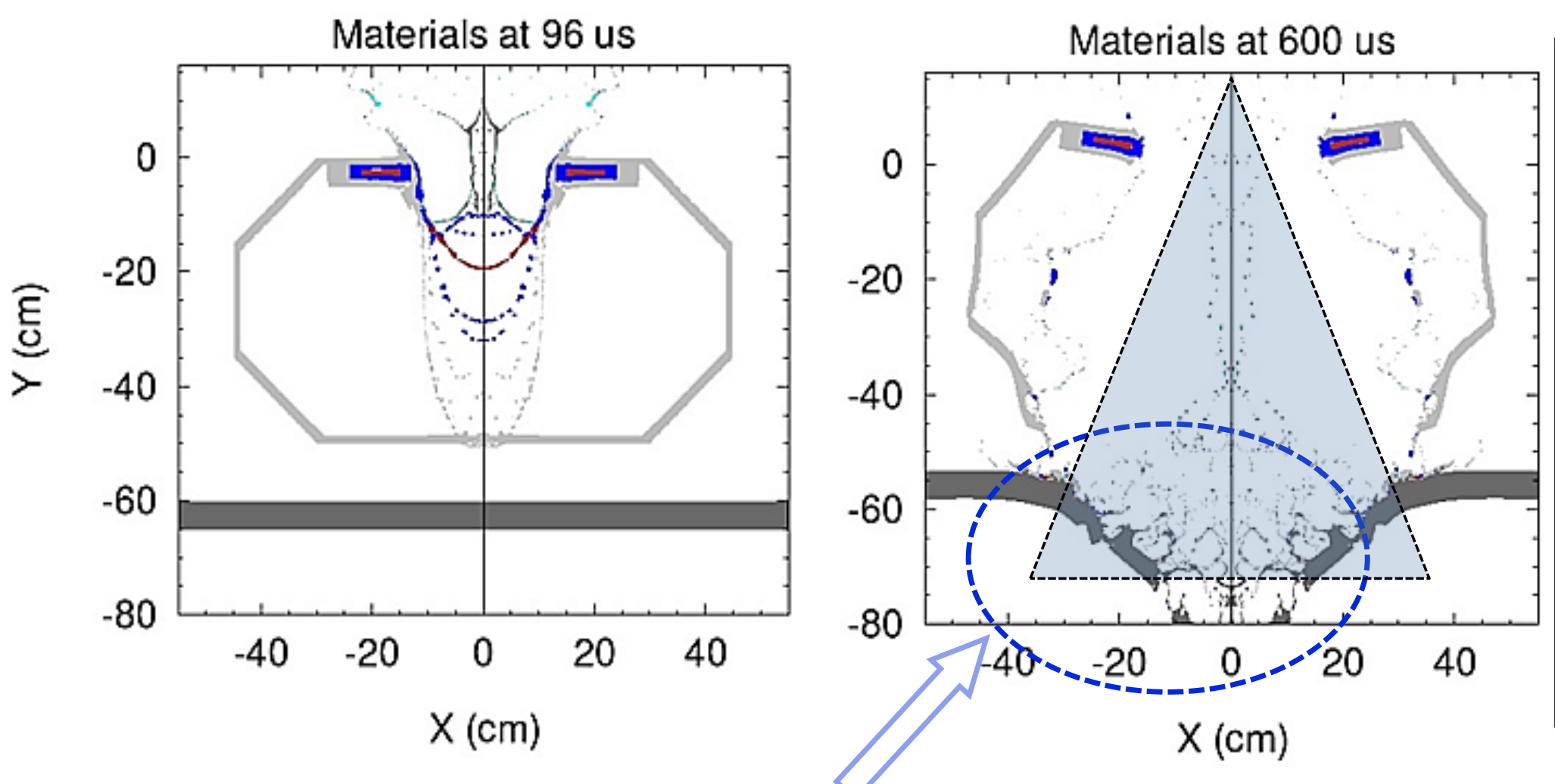

- Discrete impact location

- Discrete, primary fragments are the only mechanism to transmit damage through extended structure

- Primary fragment cone (PFC), $\sim 70^{\circ}$ total angle

CRATERING AND DISINTEGRATION CONSISTENT WITH DEBRISLV OBSERVATIONS

- Condensed Phase (i.e., solid/liquid metal fragments) "Cratering"

- Consistent with observed destruction primarily confined within PFC

- (But: No vapor/plasma phase) 


\section{Hydrocode and Primary Fragment Cone (PFC)}

Hydrodynamic Modeling for Hypervelocity Impact Fragmentation

- Hydrocode : CTH (Sandia Hydrodynamic code)

- Material Equations: Johnson-Cook Fracture Model and Grady-Kipp Dynamic Fragmentation Model (no vapor/plasma models are available)

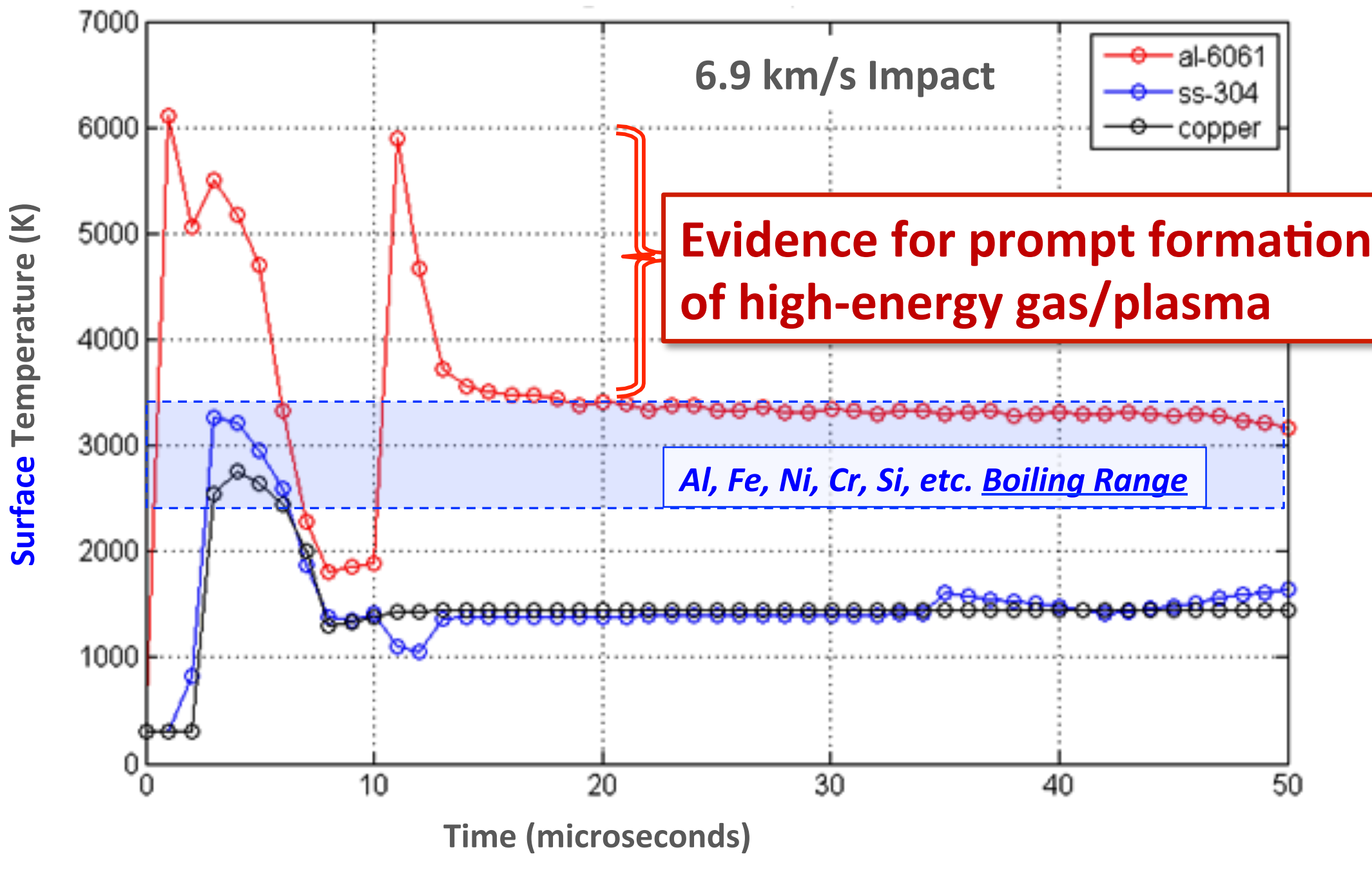




\section{DebrisLV Mounted in Soft-Catch Foam-Lined Chamber}

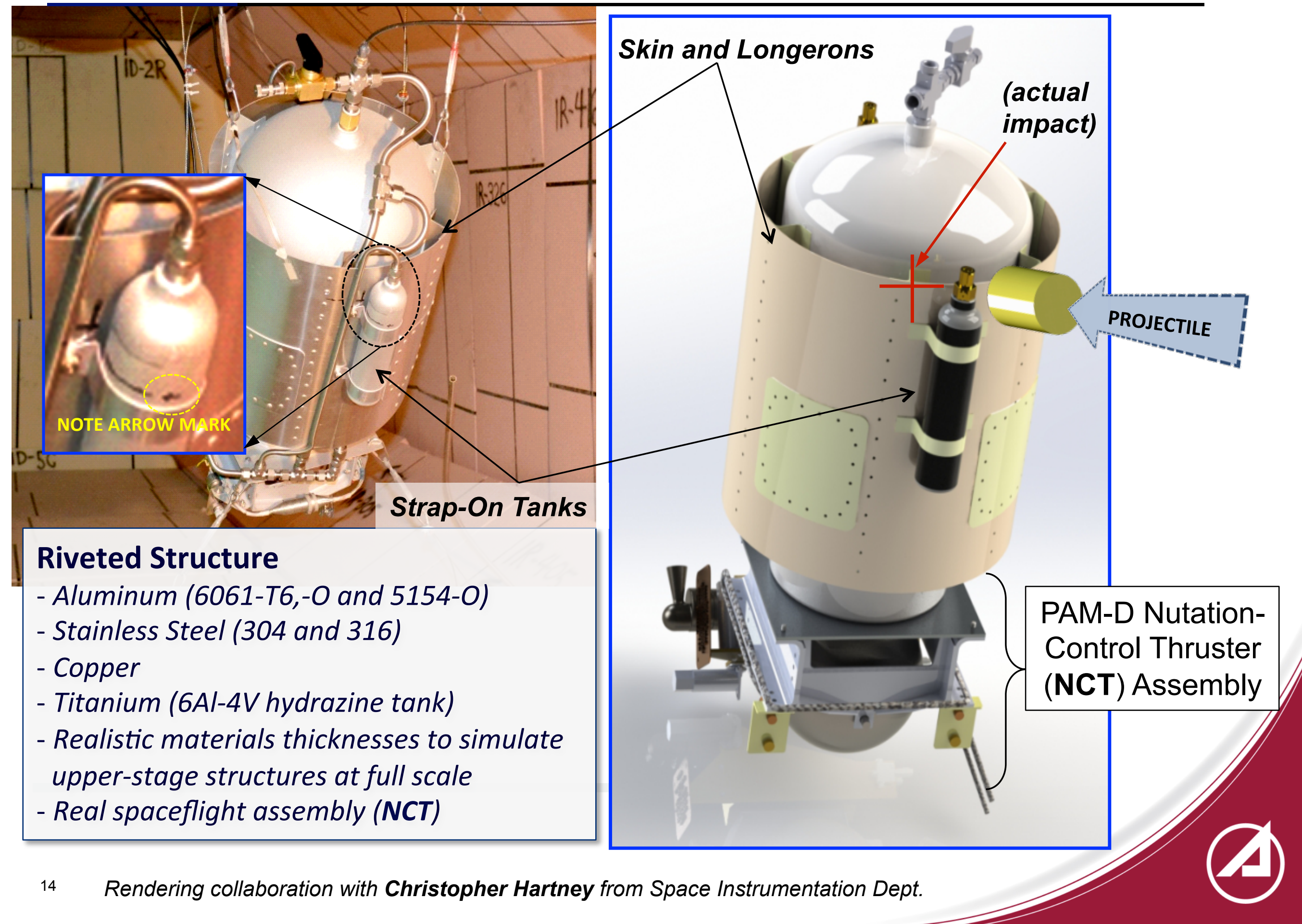




\section{Primary Fragment Cone - Side View}

\section{Compare with Hydrocode Result (slide \#12)}

Side View
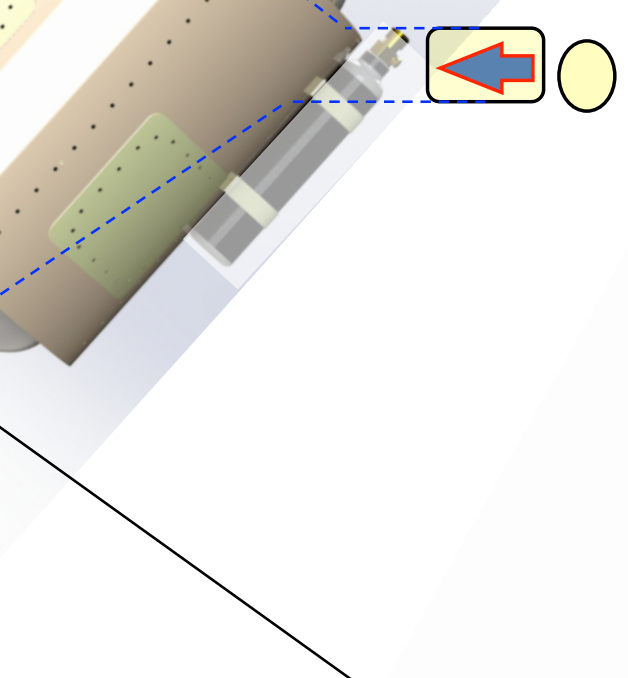

(BOTTOM)

FOOTER

\section{(TOP)}

FILL TUBE

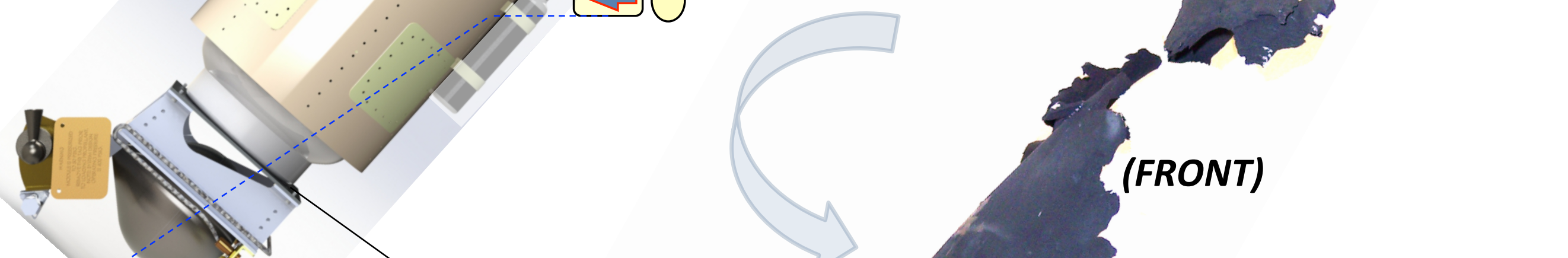




\section{Primary Fragment Cone - Top View}
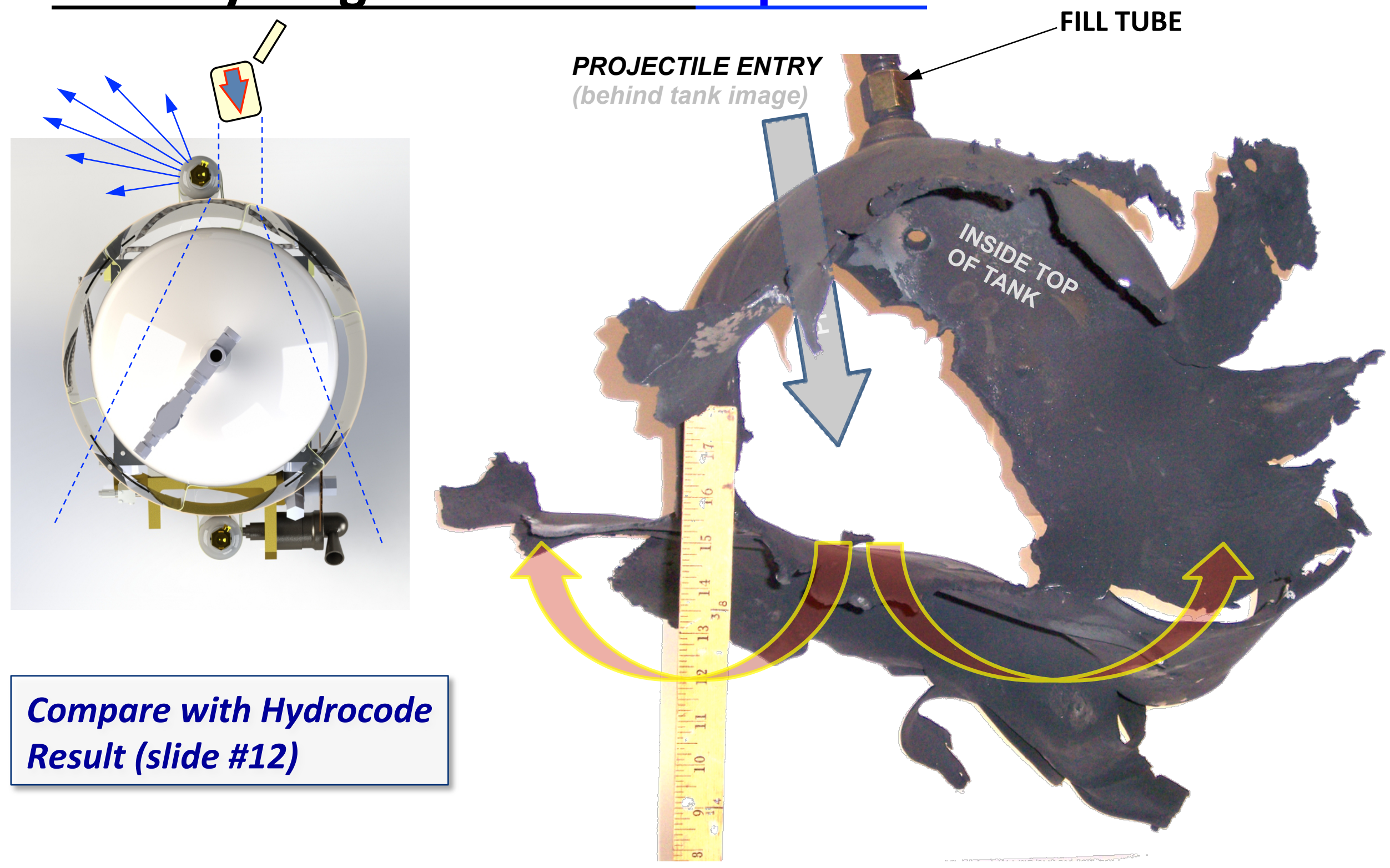

Compare with Hydrocode Result (slide \#12)

Top View 


\section{Solid- and Liquid-Phase Primary Impact Cratering}

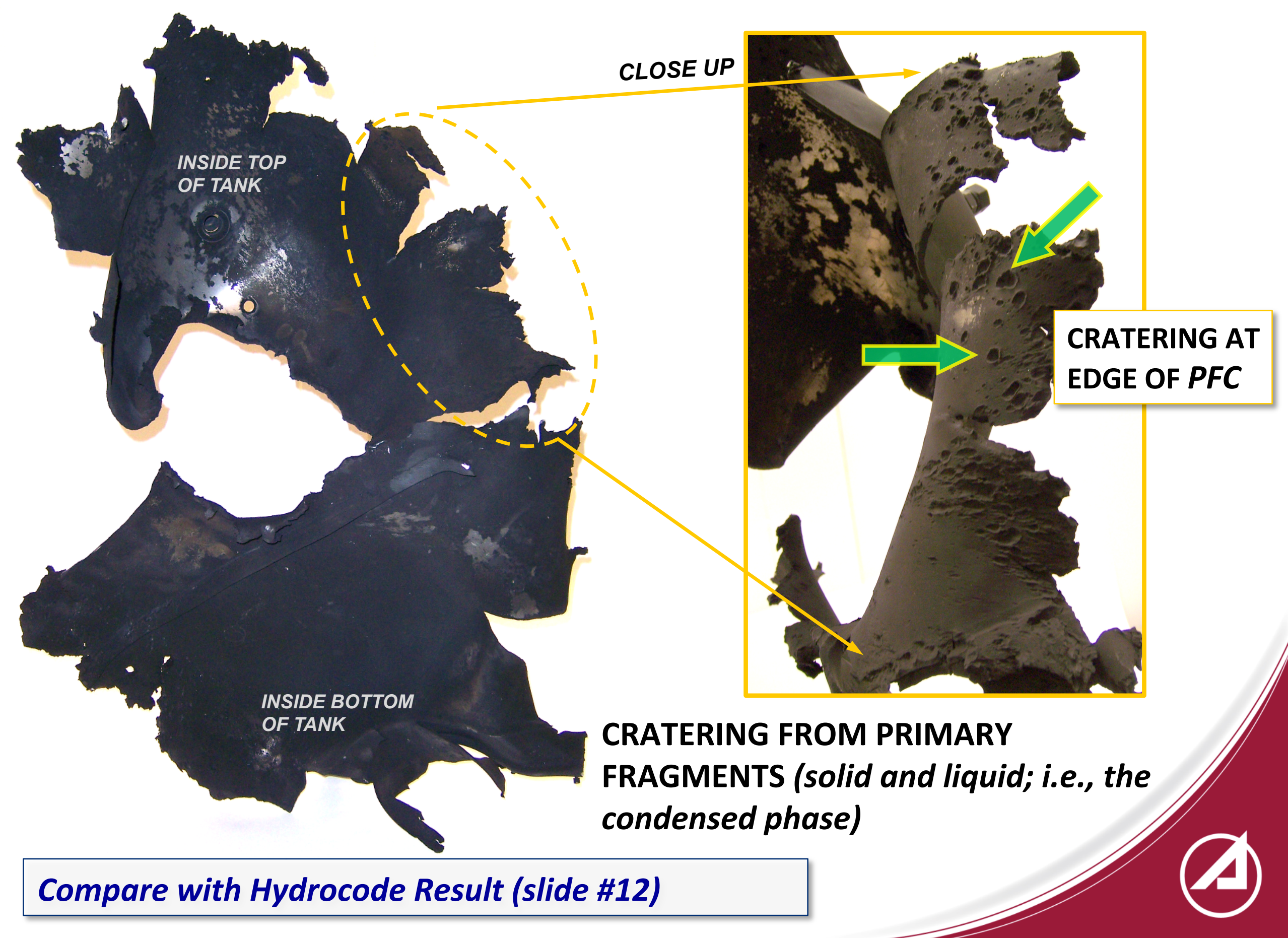




\section{Solid-and Liquid-Phase Cratering Predicted By Hydrocode}

\section{DebrisLV Main Tank Post-Shot}

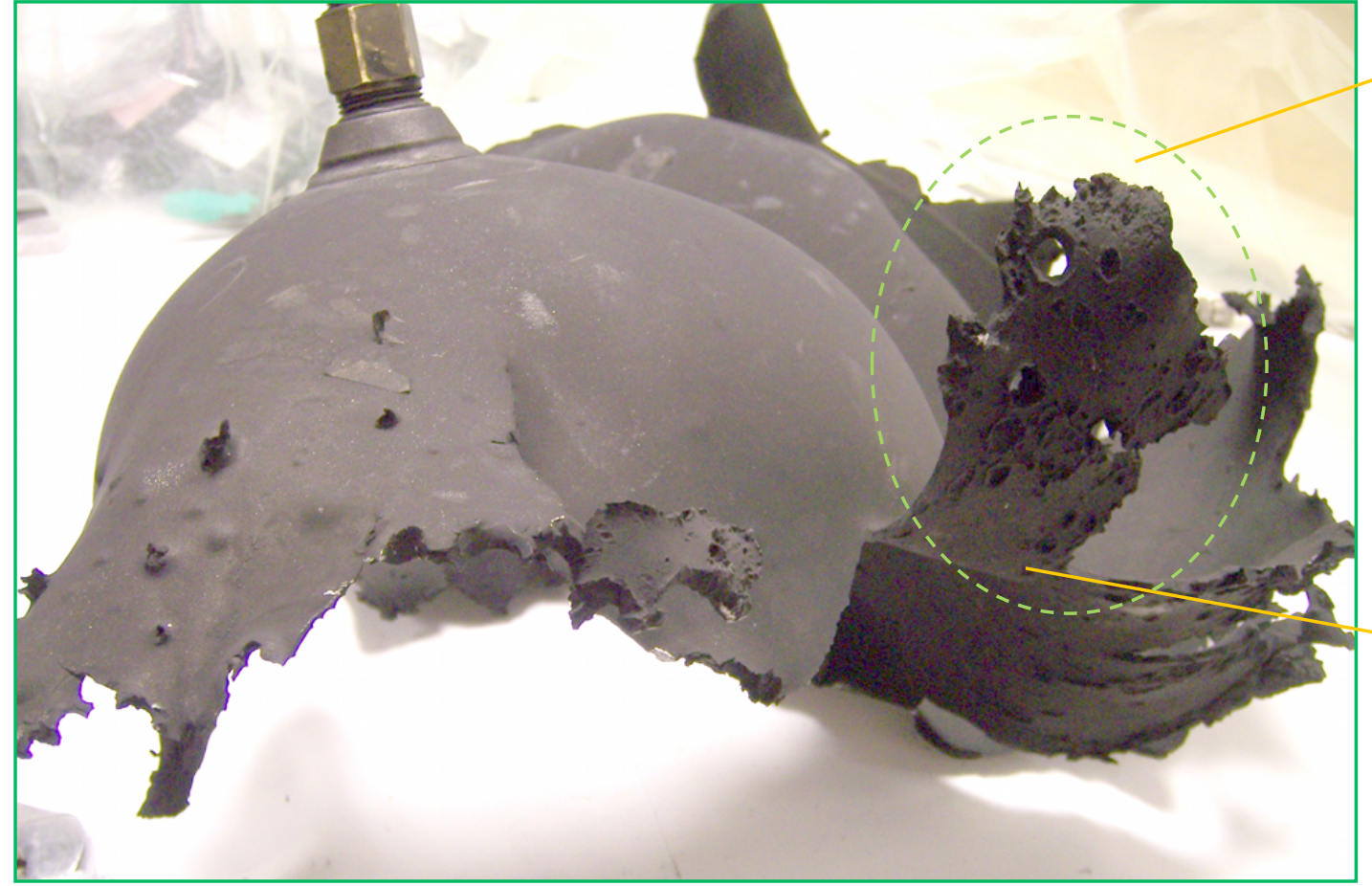

12 inches

Cratering is characteristic of both solid fragment and liquid metal droplet impacts, consistent with the current state-of-the-art for Hydrocode results. Provides a mechanism for destruction within primary fragment cone (PFC)
CRATERING AND DISINTIGRATION

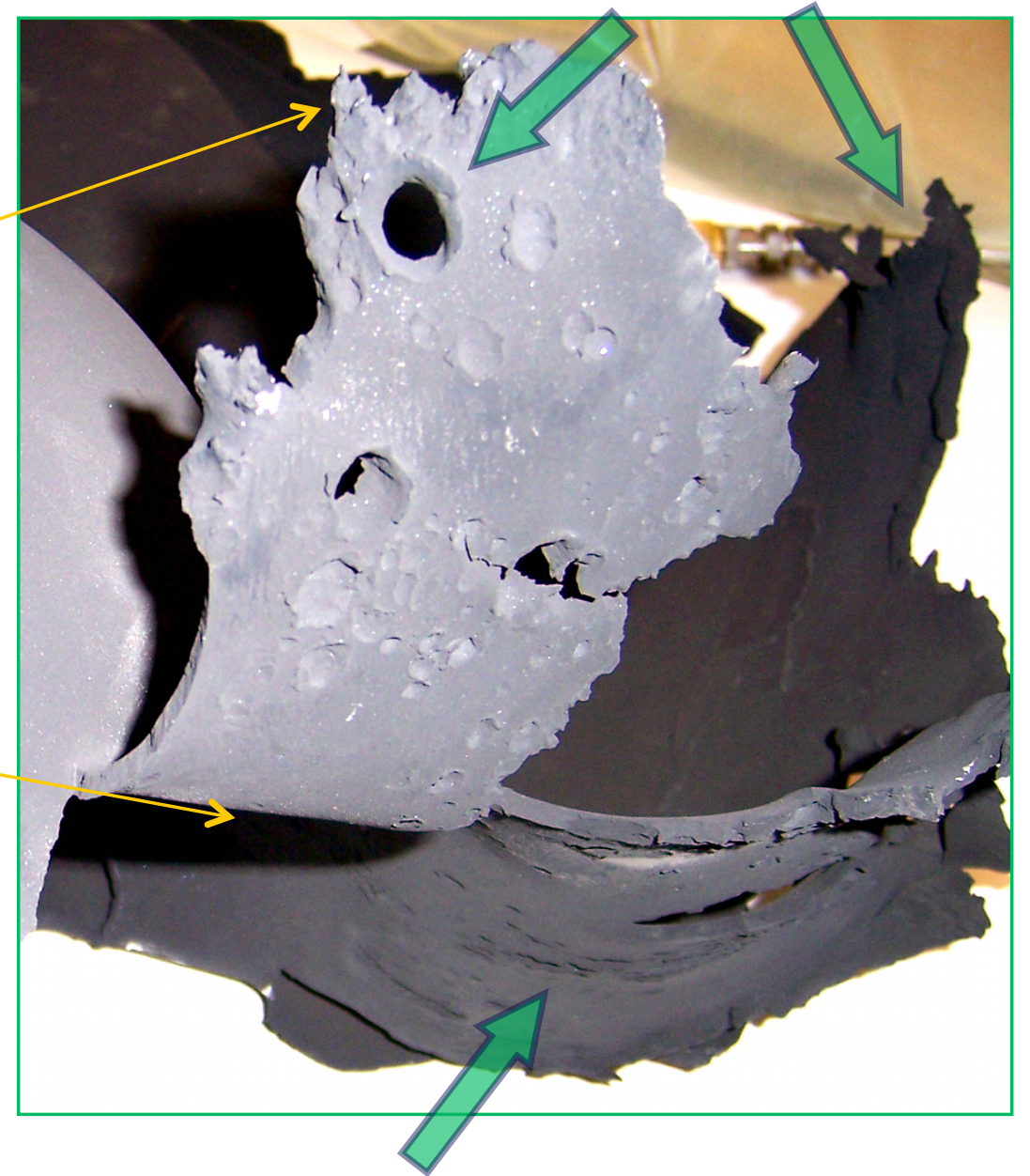

CRATERING AND DISINTIGRATION

- Defines Edge of PFC

- Solid Metal Chunks

- Liquid Metal Droplets 


\section{Evidence of Liquid Metal Condensed from Vapor Phase}

Electron Image 5

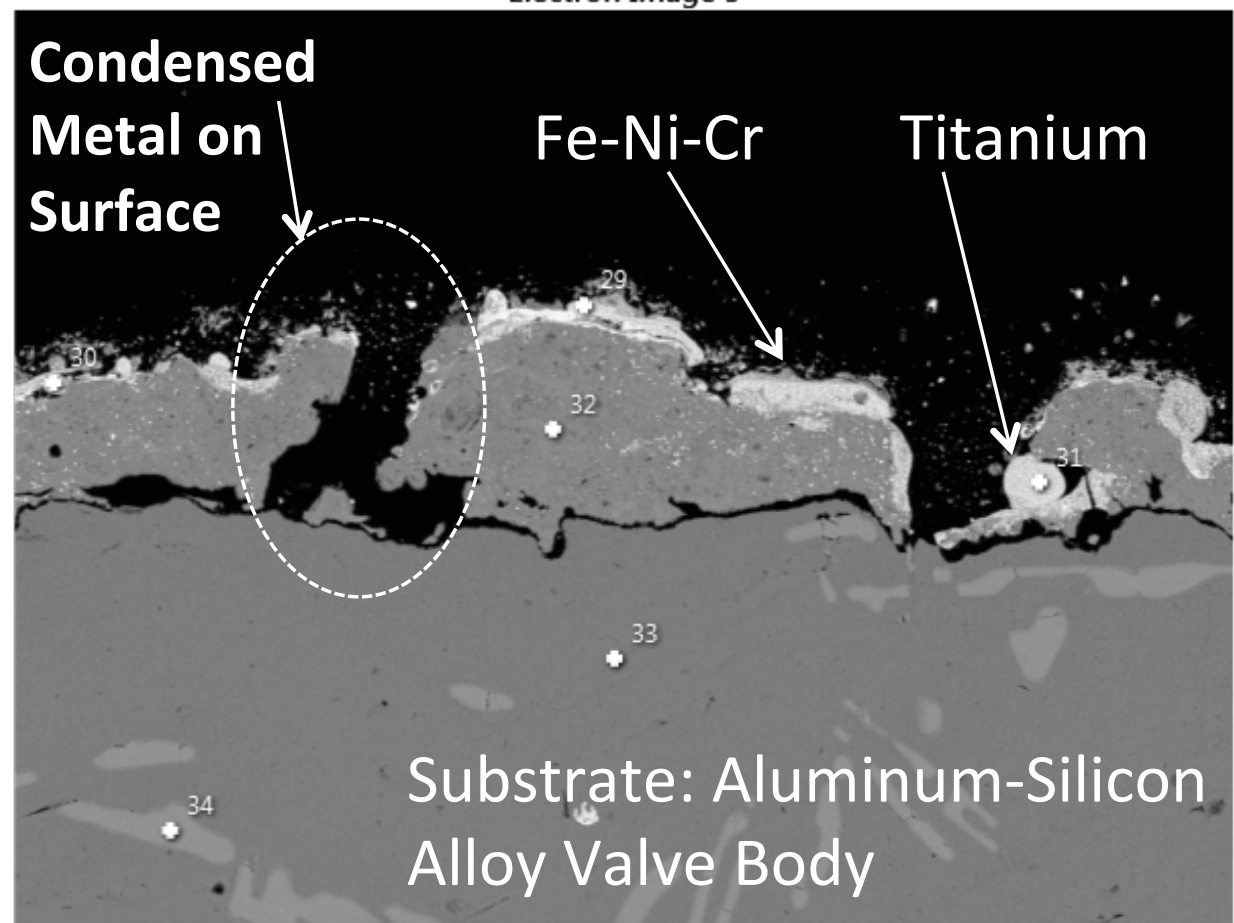

Electron Image 3

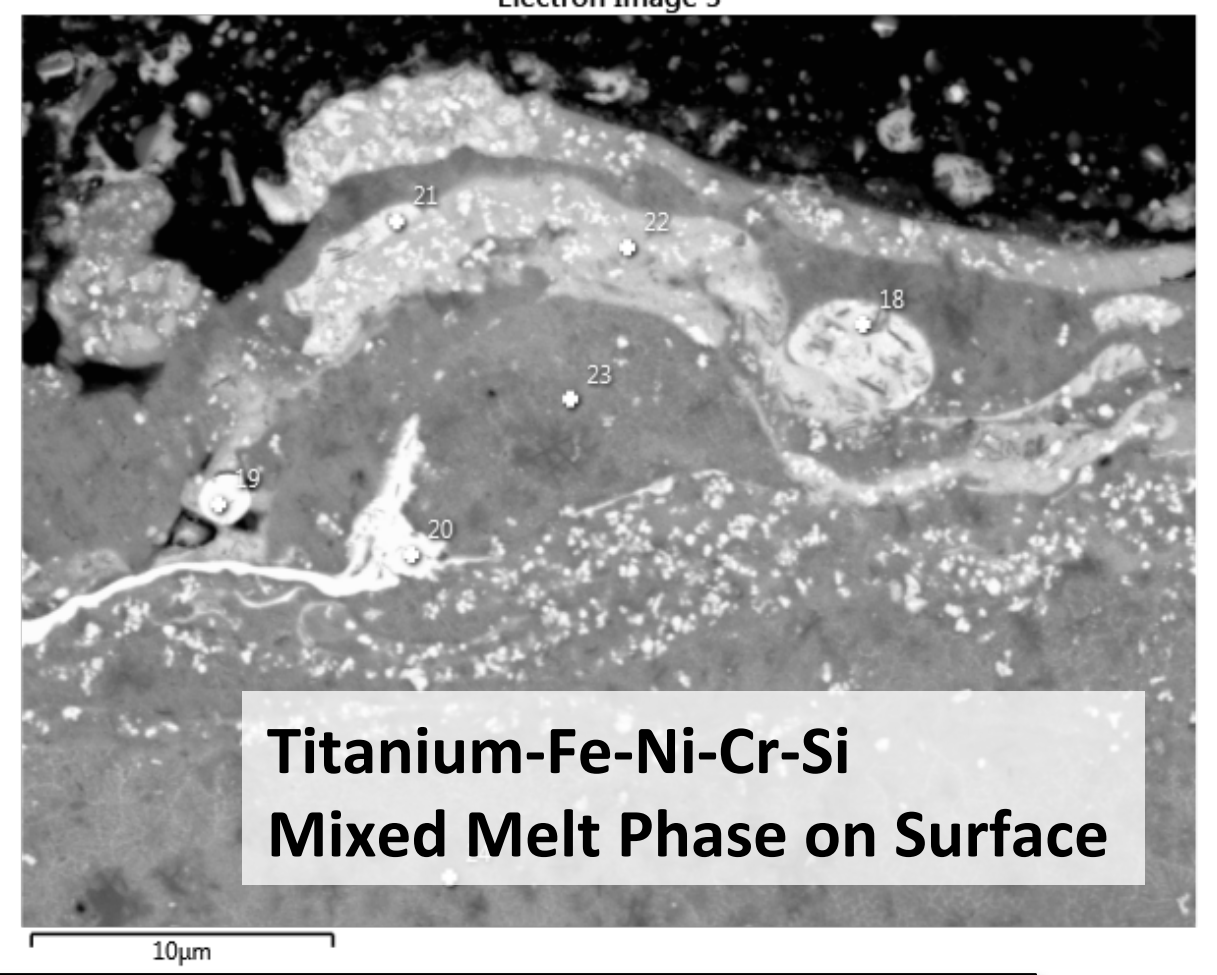

- Liquid metals do not melt and flow at these time scales

- Condensation of liquid metal from the gas phase supports several mechanisms for production of small-to-nanoscale fragments

- Solidified liquid film forms detached flakes due to thermal stresses as film cools

- Microanalysis yields evidence for gas-phase reaction and elemental segregation and molecular association (e.g., AlO formation) prior to deposition [6, 12]

- Unknown ratio of gas-phase nanoparticle nucleation versus liquid-metal vaporcondensation followed by blow-off (microparticles) 


\section{DebrisLV LAYOUT IN TEST CHAMBER}




\section{Arena and DebrisLV Appearance Before and After Shot}

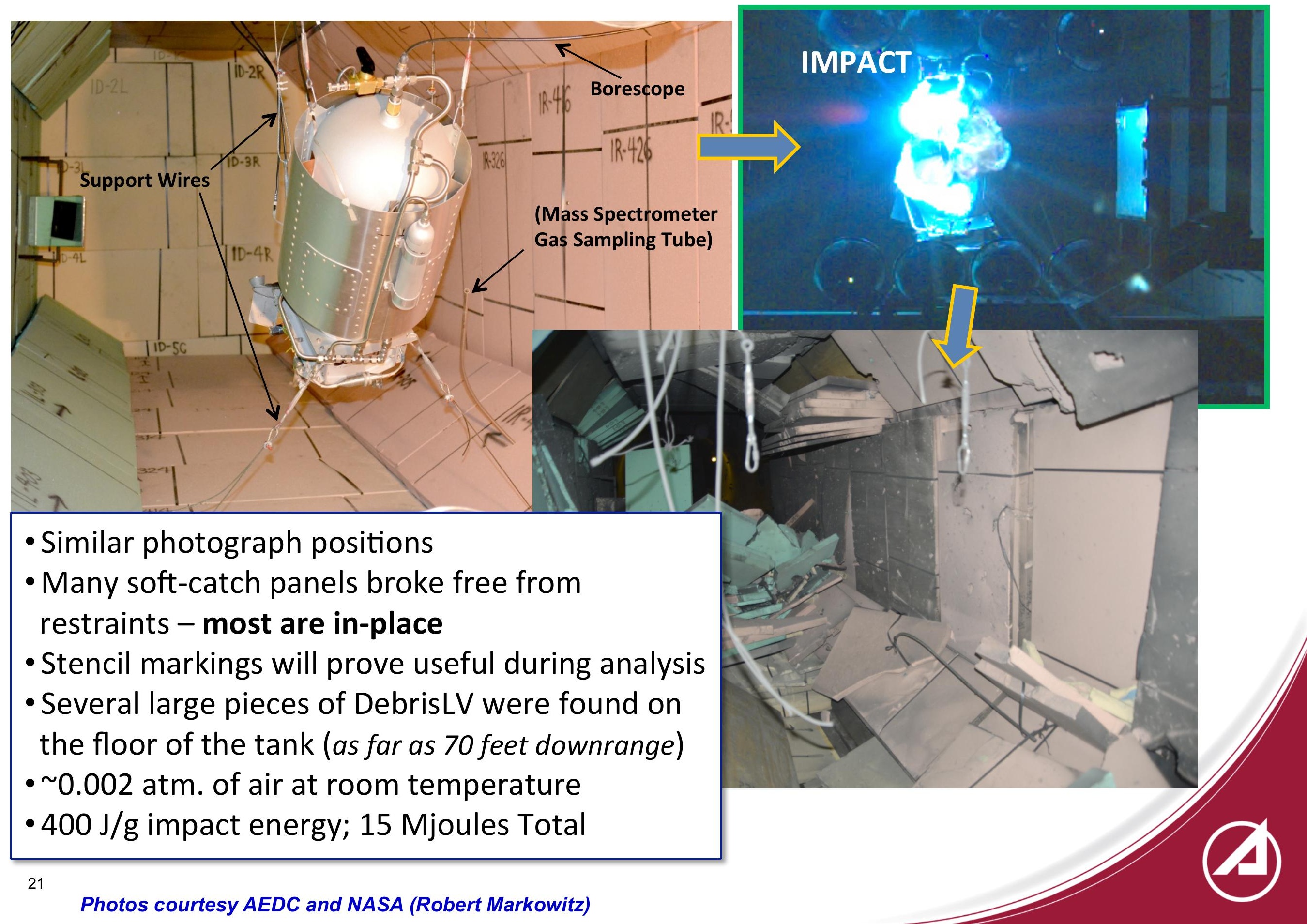




\section{DebrisLV Subassemblies}

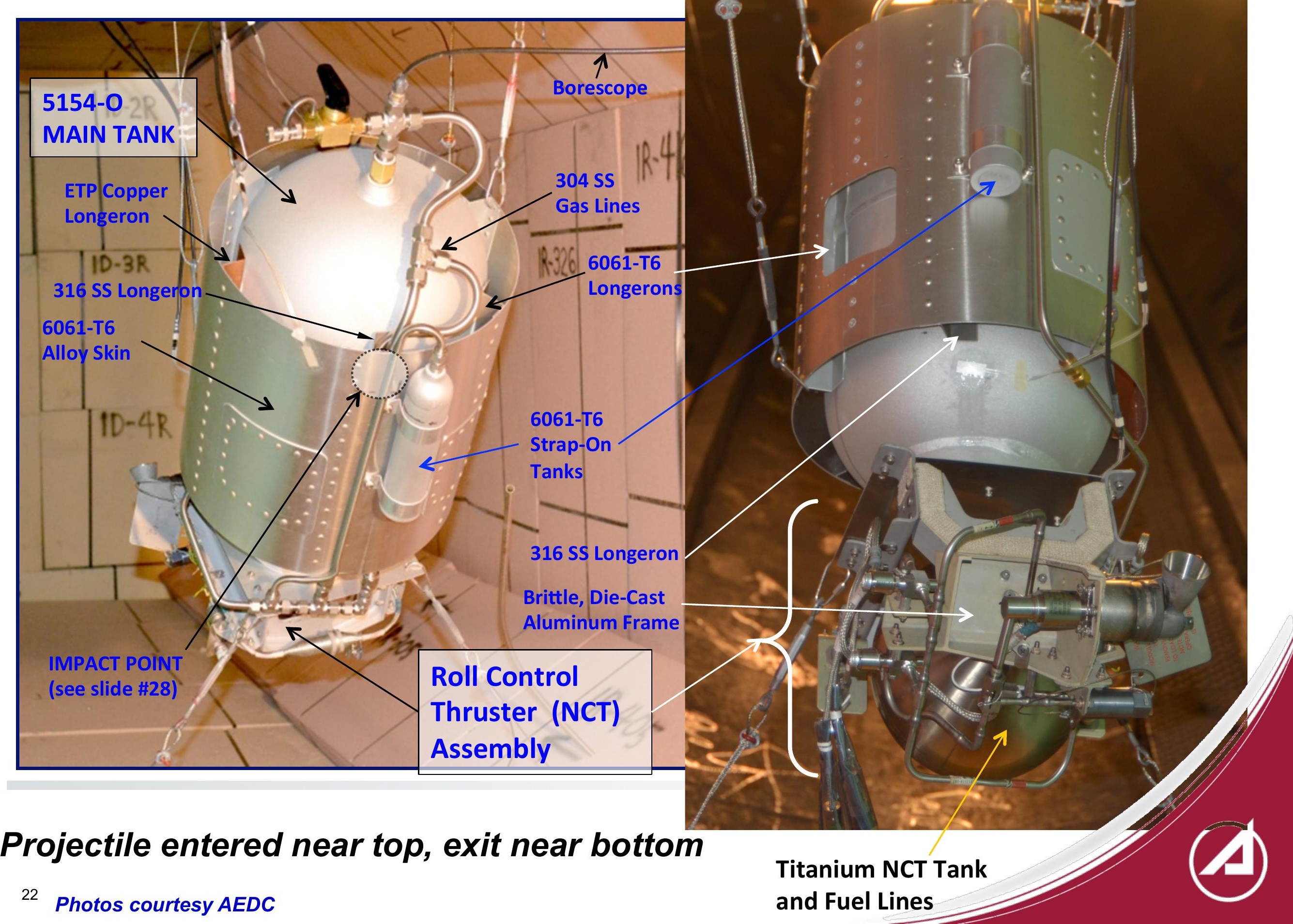




\section{DebrisLV Projectile Design (AEDC)}

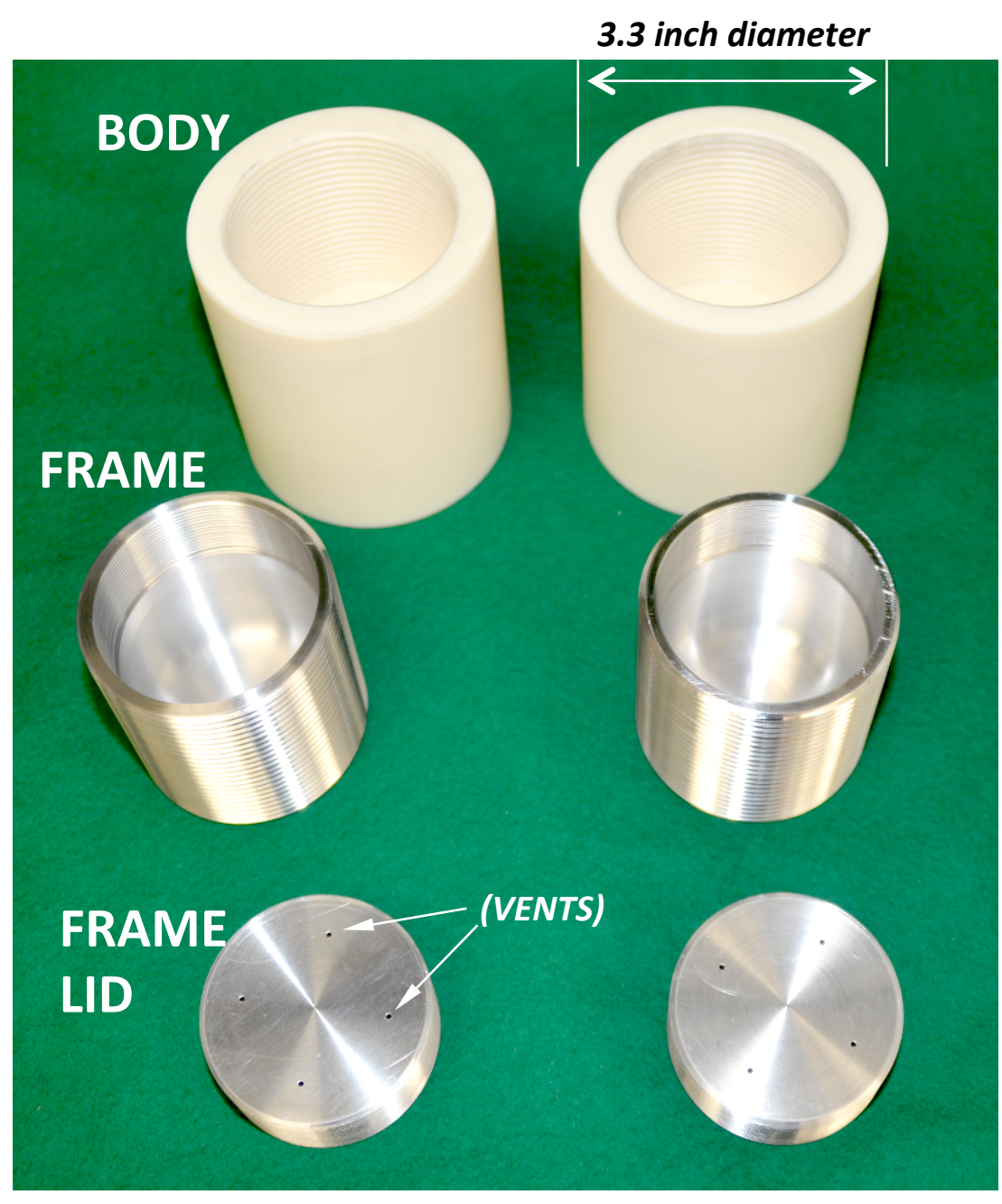

- 6061-T6 Frame

- Nylon Body

- Hollow Center for Electronics

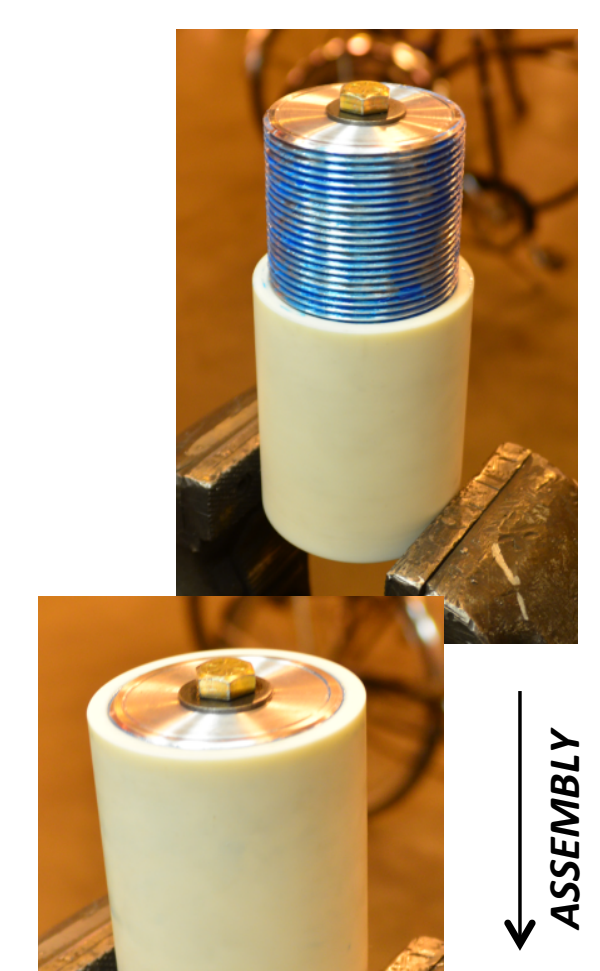

- Designed hollow for peak speed $(\sim 7 \mathrm{~km} / \mathrm{s}) ; \sim 3.3$ in. dia $x \sim 5$ in. tall

- Hollow, heavy-walled design effectively simulates a nonsolid object such as debris or a miniature satellite

- Nylon tail-piece of penetrator detached during acceleration, effectively simulating a solar-panel

- 15 MJoules energy

Projectile models miniature satellite or fragment-like object. 


\section{$\underline{\text { X-Ray Photographs of Projectile in Flight }}$}

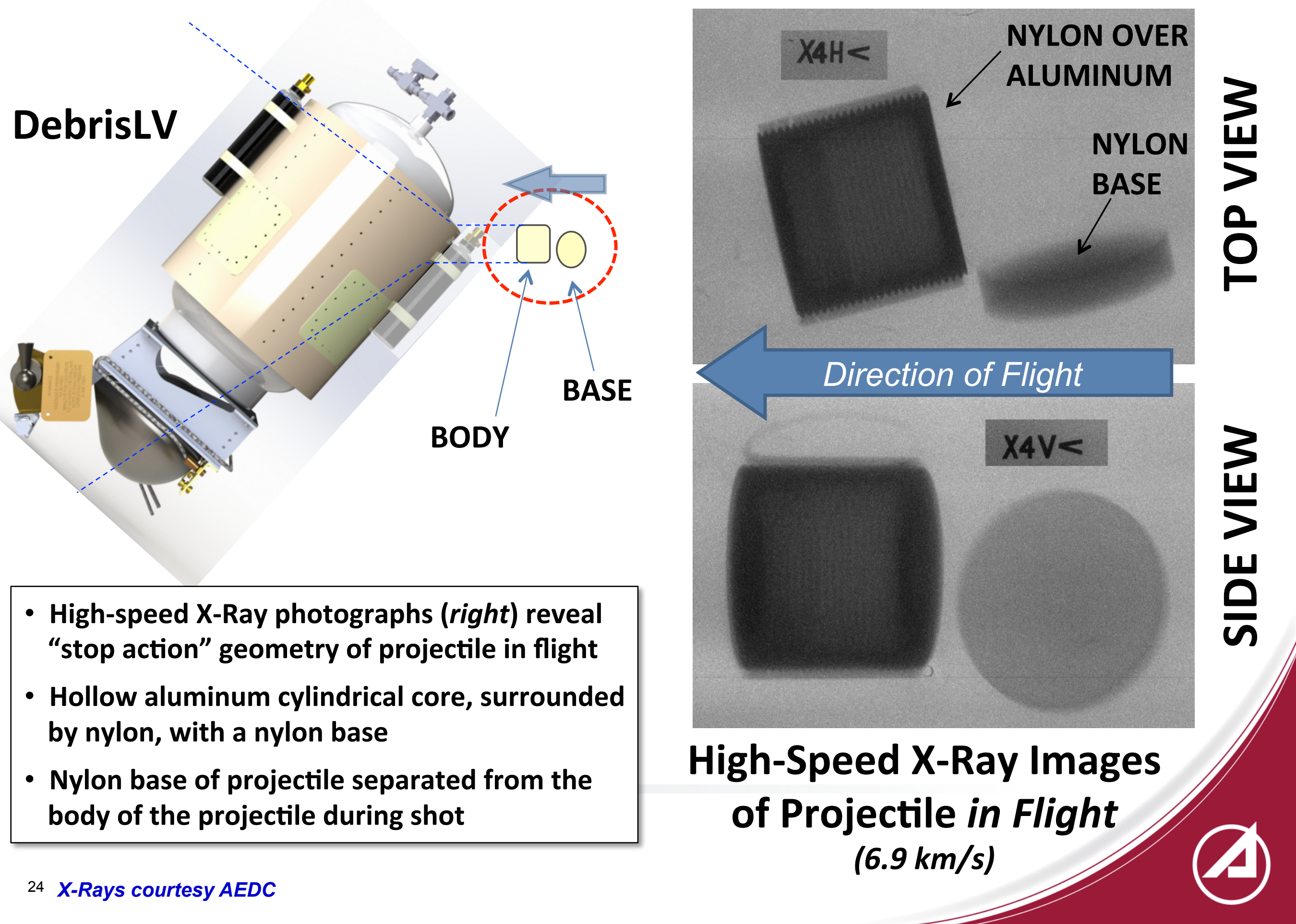




\section{Projectile Flight Path for DebrisLV}

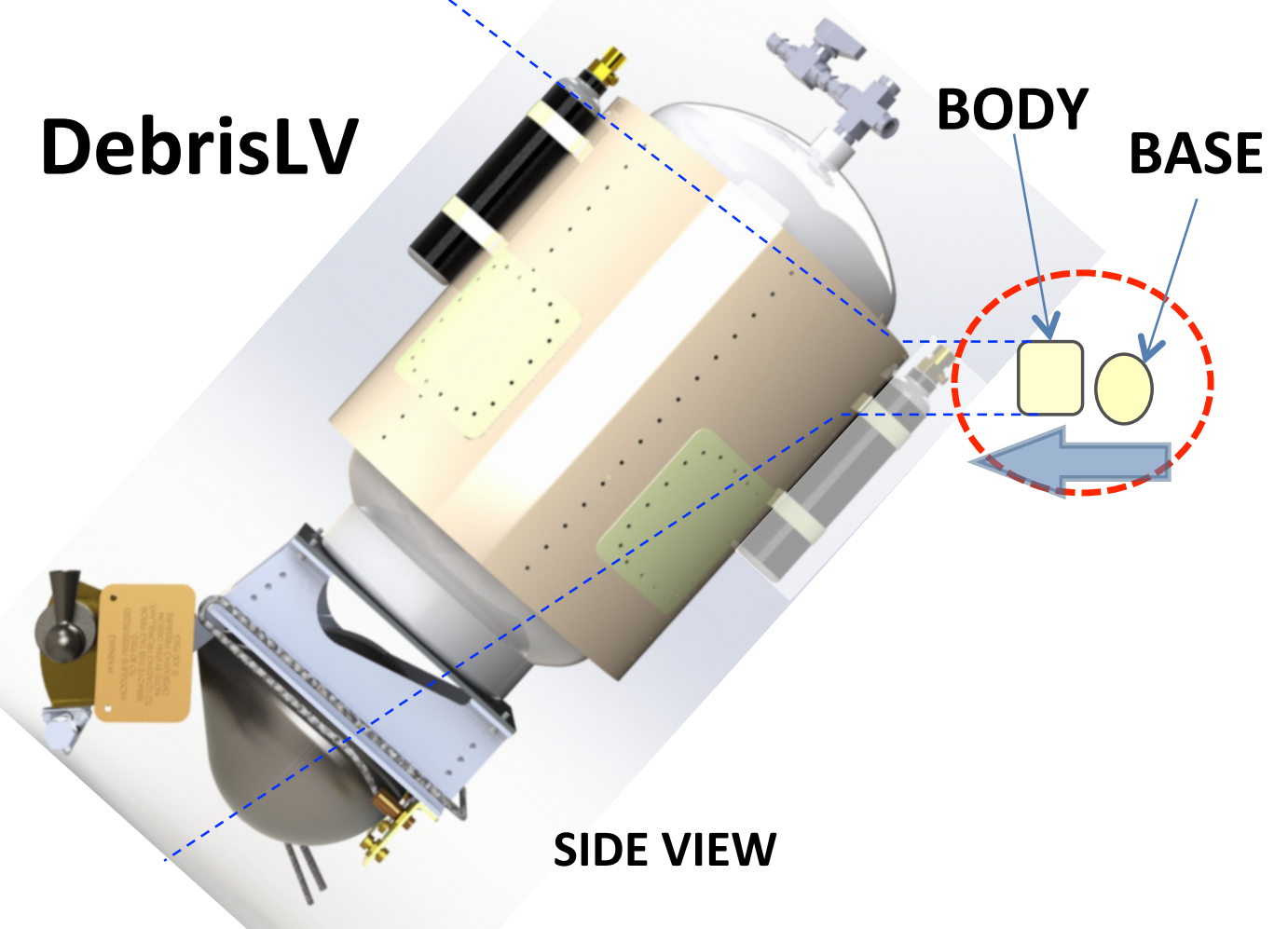

- $\sim 600 \mathrm{~g}$ projectile; $\sim 7 \mathrm{~km} / \mathrm{s}$

- $\sim 3.3$ inch diameter $x \sim 4$ inch long

- 15 psia xenon in main tank; 50 psia helium in NCT titanium tank

- Tank mounted $\sim 45^{\circ}$ angle; projectile flying horizontally as shown (left)

- Approximate Primary Fragment Cone (PFC) shown in blue dashed lines

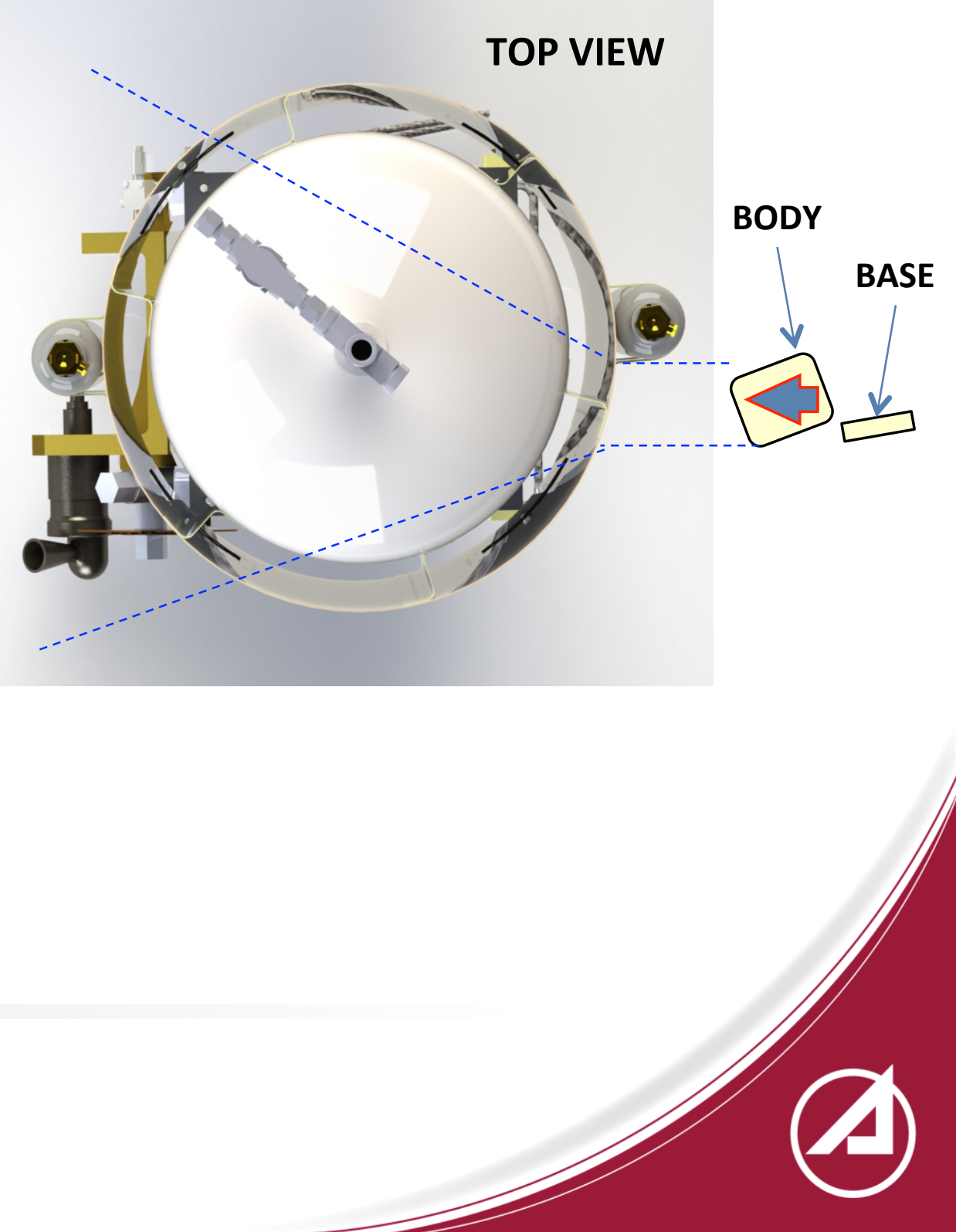




\section{AEDC Main Vacuum Chamber: View From Gun Muzzle}

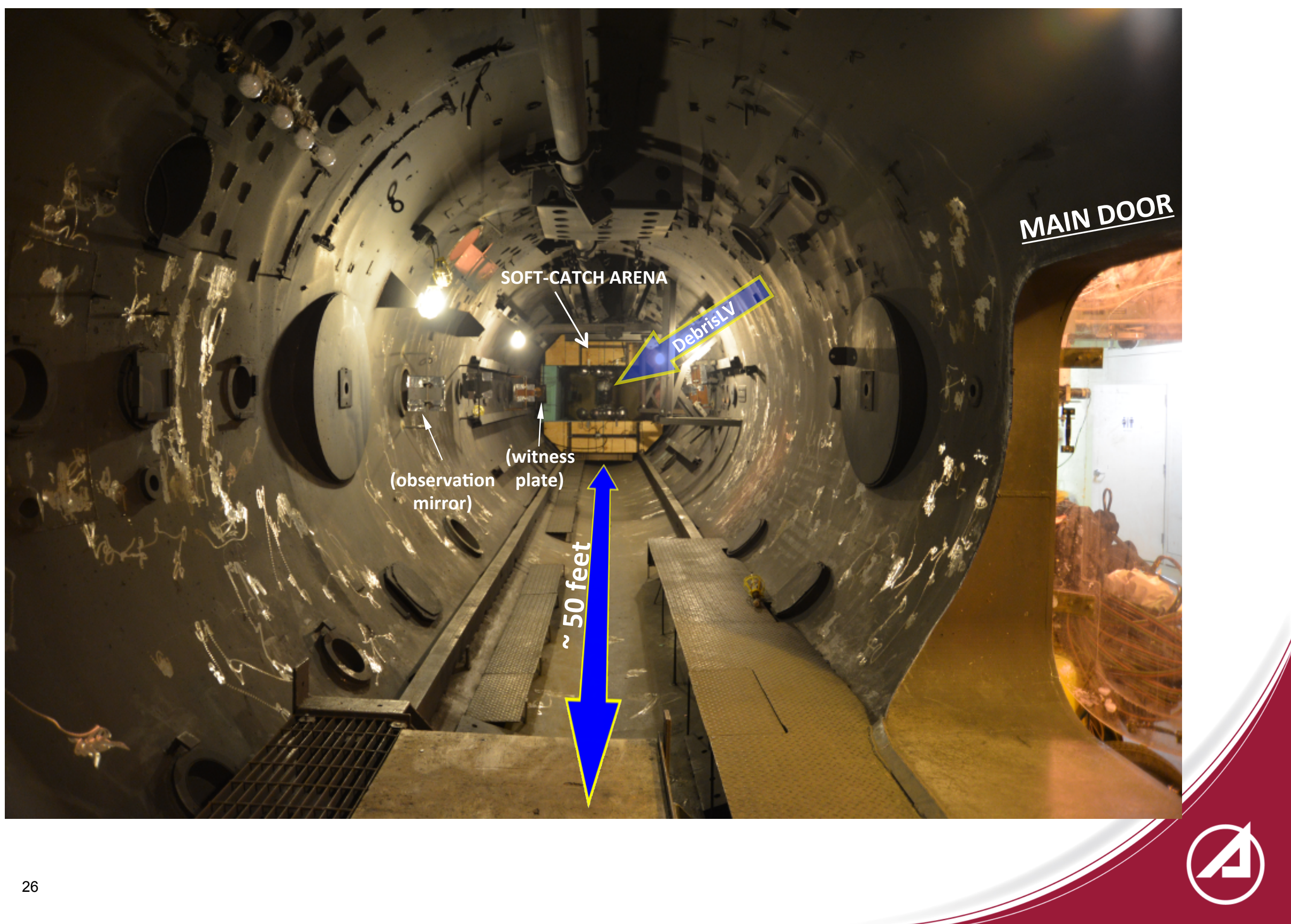




\section{GAS/PLASMA CLOUD BLAST PATTERN}

Observed plasma-jets are an unexpected, limited-range mechanism for the partitioning of energy through complex space structures.

- A limited-range fragmentation mechanism has been needed in order to explain fragmentation processes observed on-orbit.

Observations on retention of fasteners

- Rivets and screws in metal vs. carbon fiber composites 


\section{DebrisLV Early Blast Sequence}

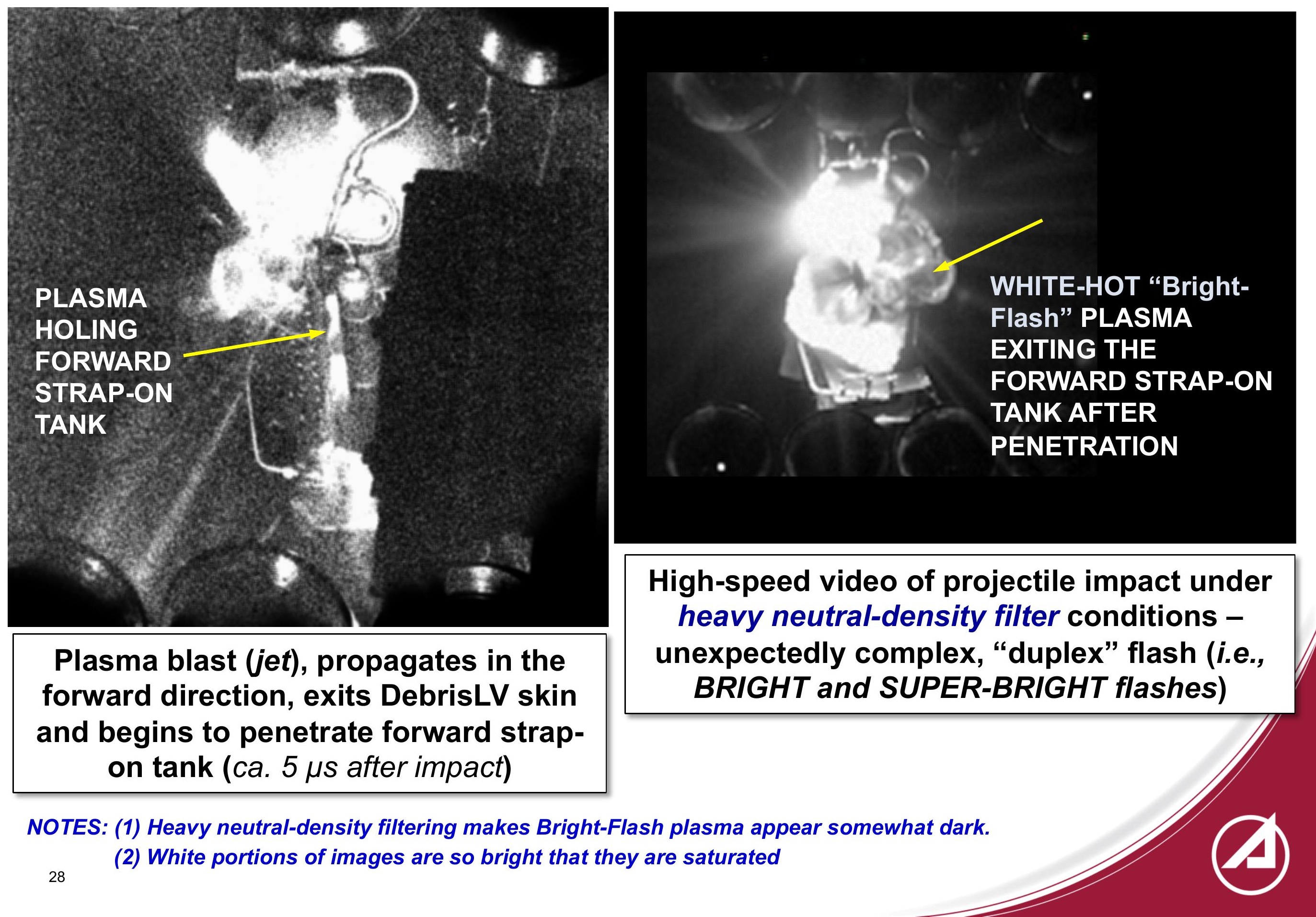




\section{Forward-Direction Metal Vapor Blast}

FORWARD STRAP-ON TANK DESTROYED BY PLASMA JET, NOT FRAGMENTS
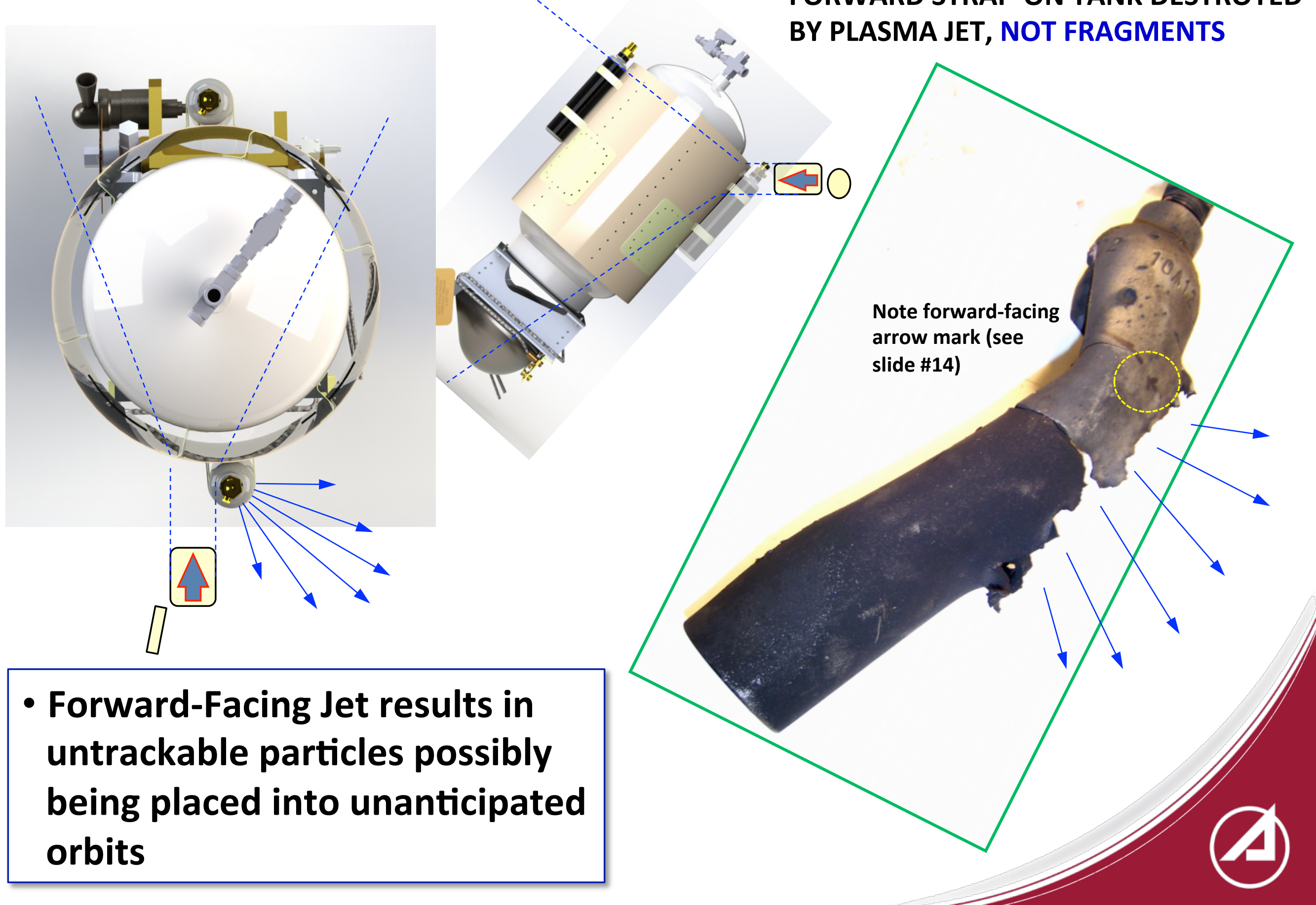


\section{DebrisLV Strap-On Tank and Plasma/Vapor Blast}

Note: Pressure tank is rated at 2,000 psi

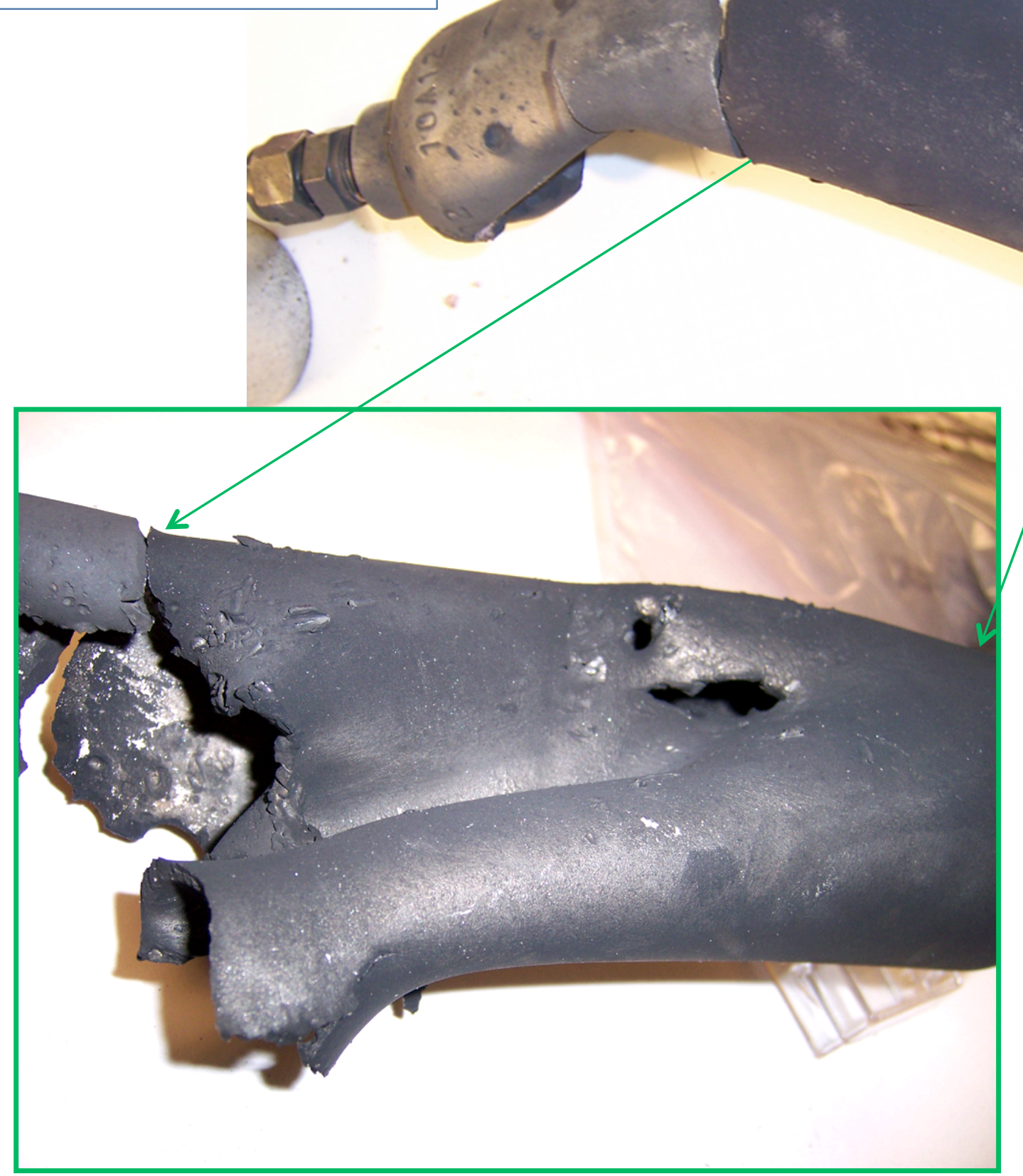

$>$ No cratering

$>$ Outside of PFC

$>$ Metal vapor Jet only

$>$ Sliced through, and deeply distorted, a very stout tank!

$>$ Inside coated with aluminum 


\section{DebrisLV Strap-On Tank and Plasma/Vapor Blast}

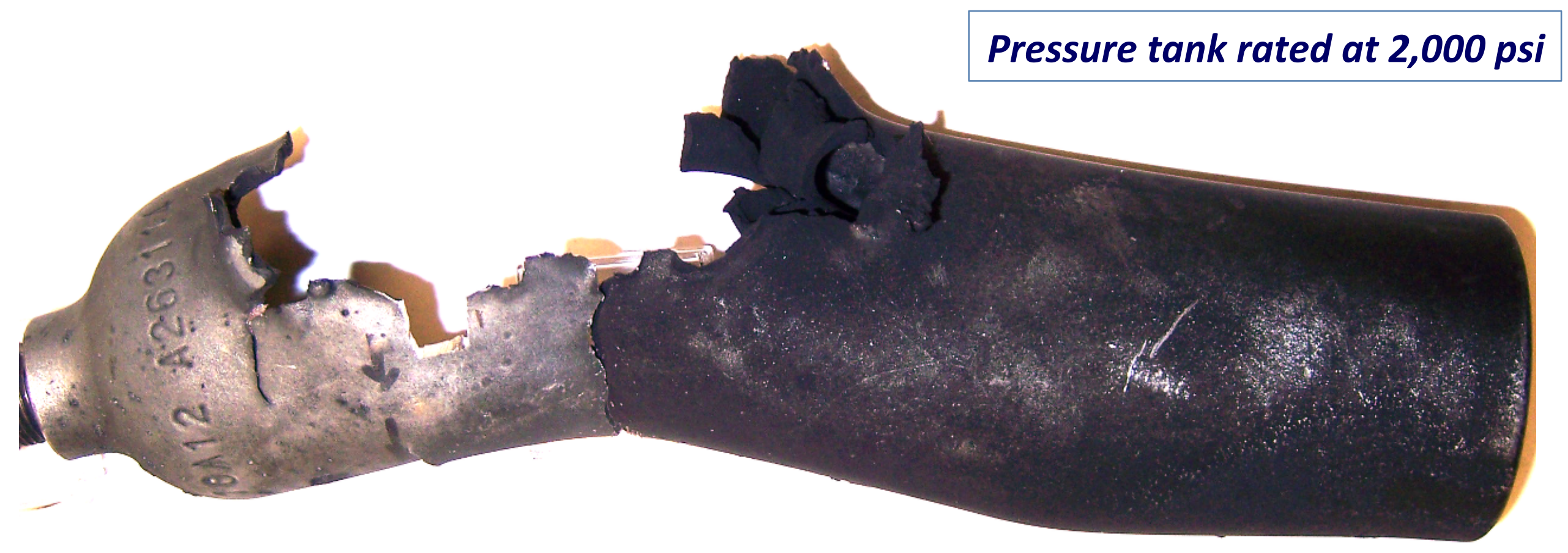

No cratering observed because tank was located outside of PFC

$>$ Metal plasma/vapor jet was only energy transfer mechanism

$>$ Sliced through, and deeply distorted, a very stout tank!

$>$ Inside coated with aluminum condensed metal from metal jet 


\section{DebrisLV vs. DebriSat Duplex Plasma Timelines}
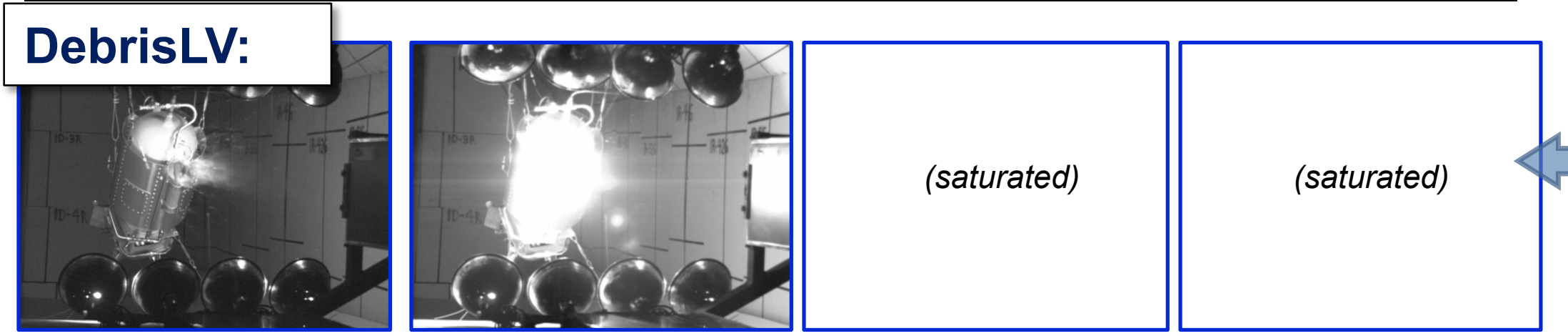

Normal AEDC Exposure Profile
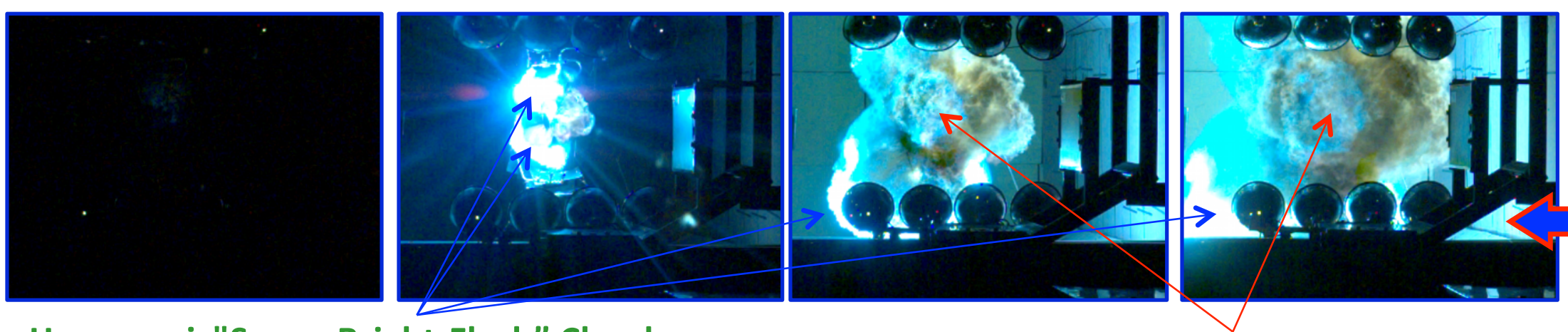

Aerospace Exposure Profile

Hypersonic"Super-Bright-Flash" Cloud

DebriSat:

Chemistry in Turbulent, Hypersonic (but slower) "Bright-Flash" Cloud
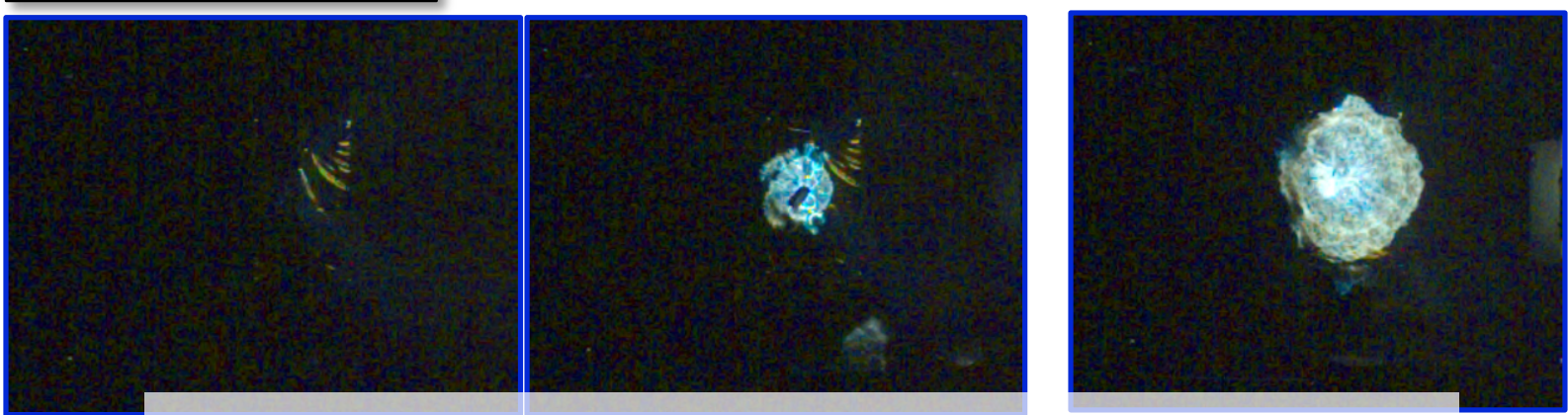

Note: NO "Super Bright-Flash" Cloud is observed in DebriSat
$-10$
$+30$
$+60$
+90 TIME (usec) +130

Different energy liberation rates suggest different fragmentation 


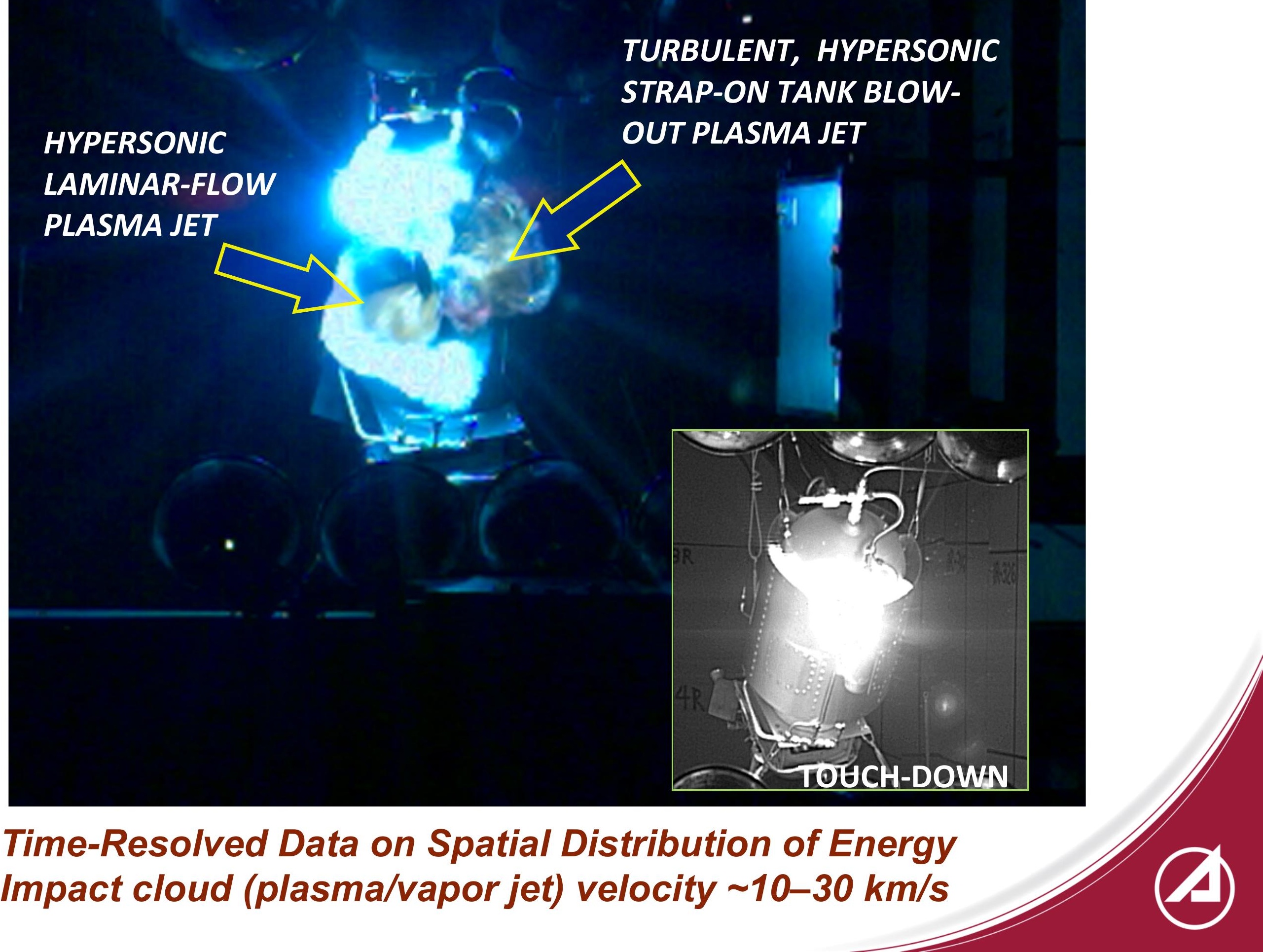




\section{DebrisLV Infrared Blast Sequence}

- Sensors on Main Vacuum Tank Exterior

- Aerospace Hypertemporal Multispectral Imager (AHMI) 4-5 $\mu \mathrm{m}$ infrared

- NASA Color Phantom Camera (Aerospace Settings to Capture Flash Temperature)
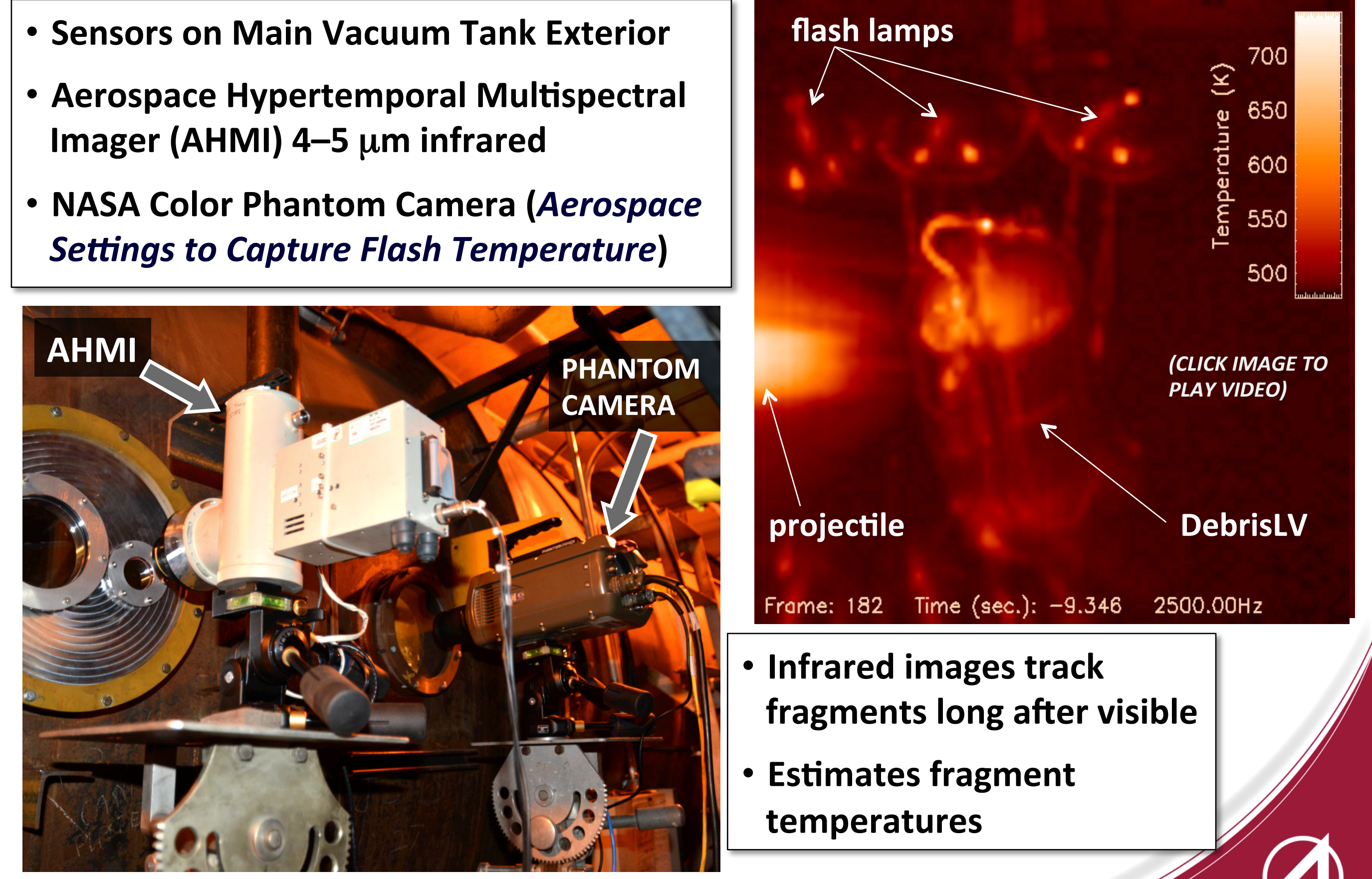

- Infrared images track fragments long after visible

- Estimates fragment temperatures 


\section{PLASMA VAPOR DEPOSITION}

- Short-range impulse transfer to surrounding structure

- Metal deposition, condensation, and flaking

$>$ New, mechanism for producing untrackable (i.e., less than $1 \mathrm{~cm}$ ) space-debris fragments 


\section{Bare DebrisLV Main Tank Before and After Shot}

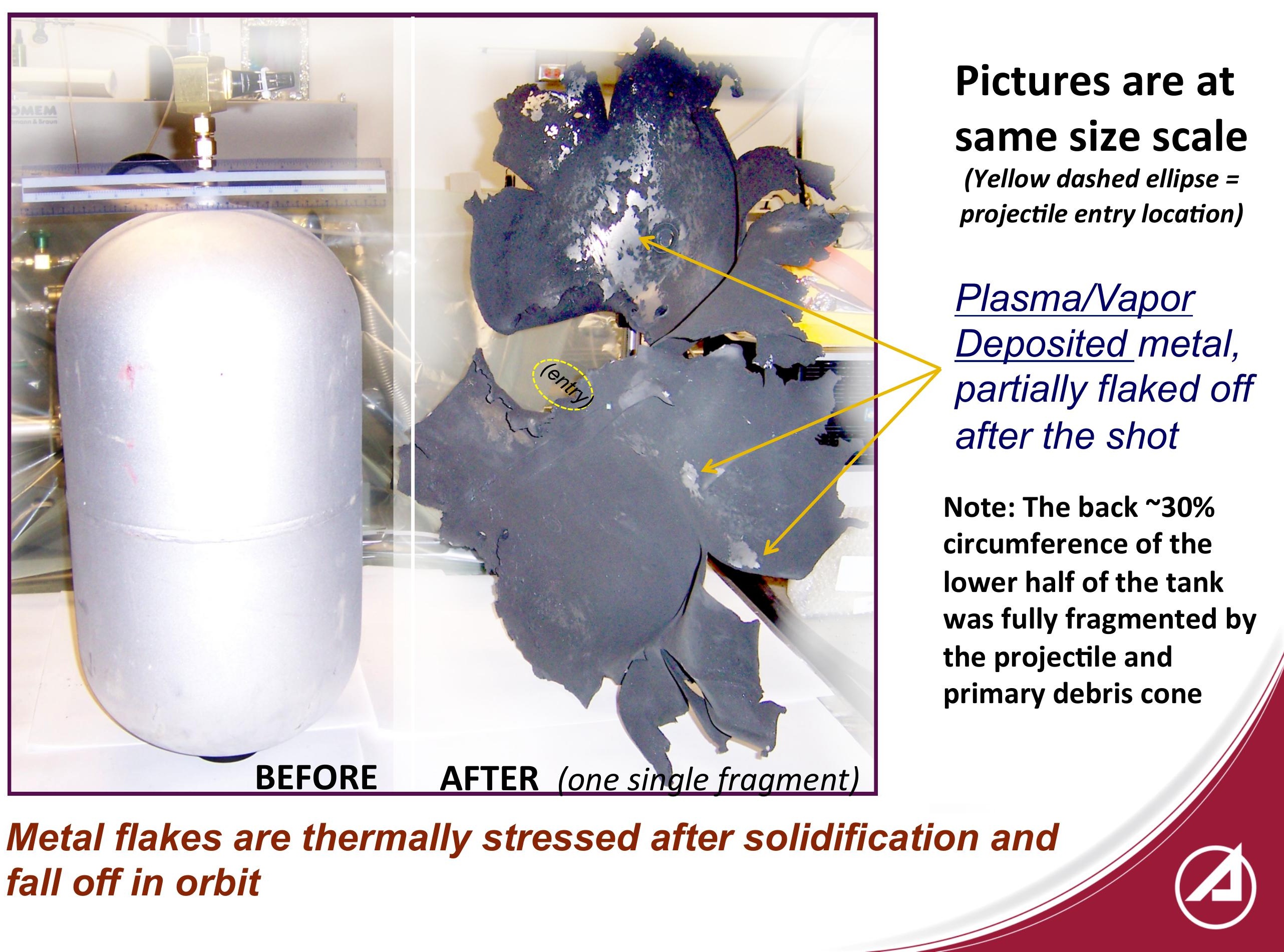




\section{NCT Titanium Tank Fragments - Plasma Jet Blast}

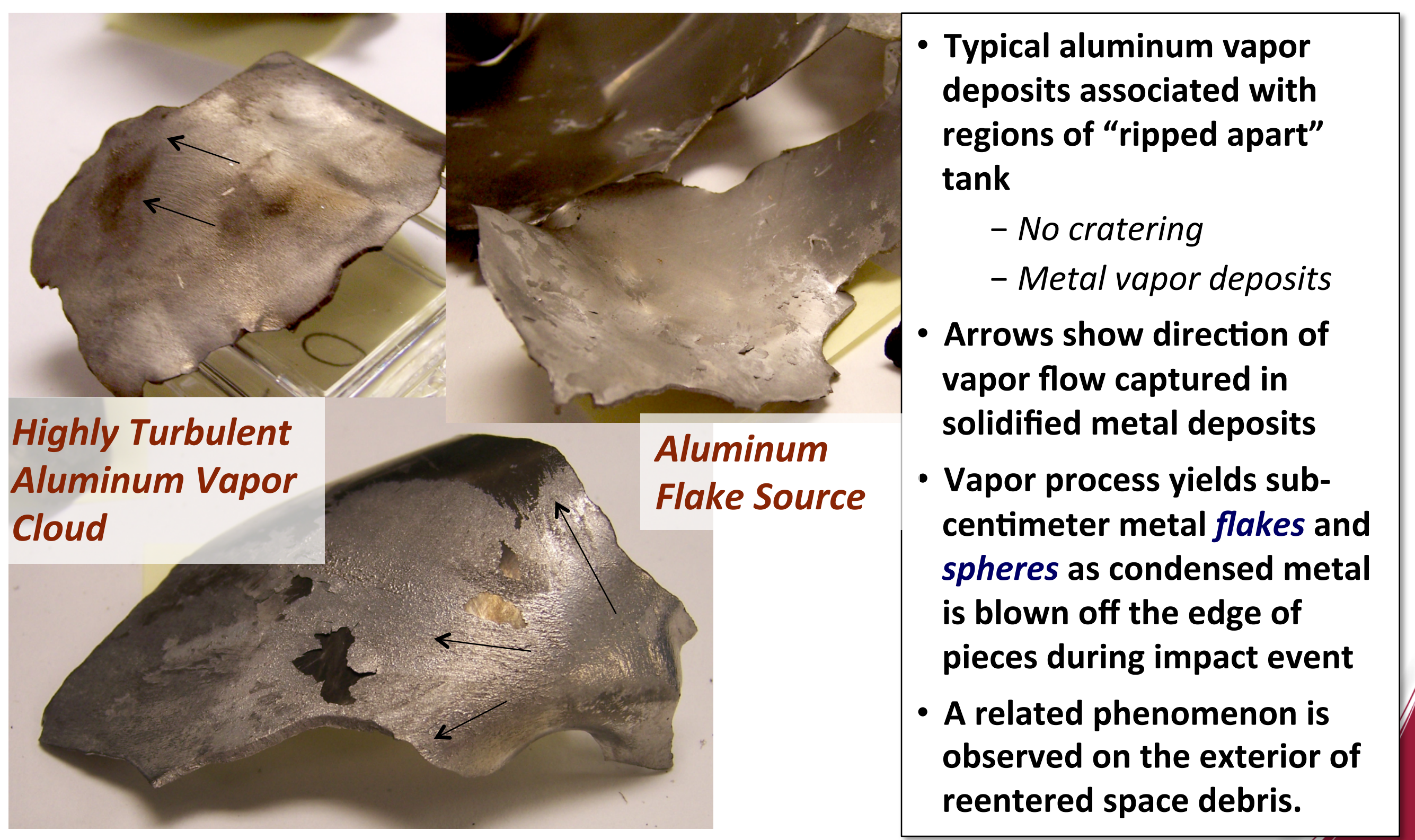

Ubiquity of aluminum vapor suggests new path to small fragments 


\section{NCT Chassis Large-Fragment Brief Survey}

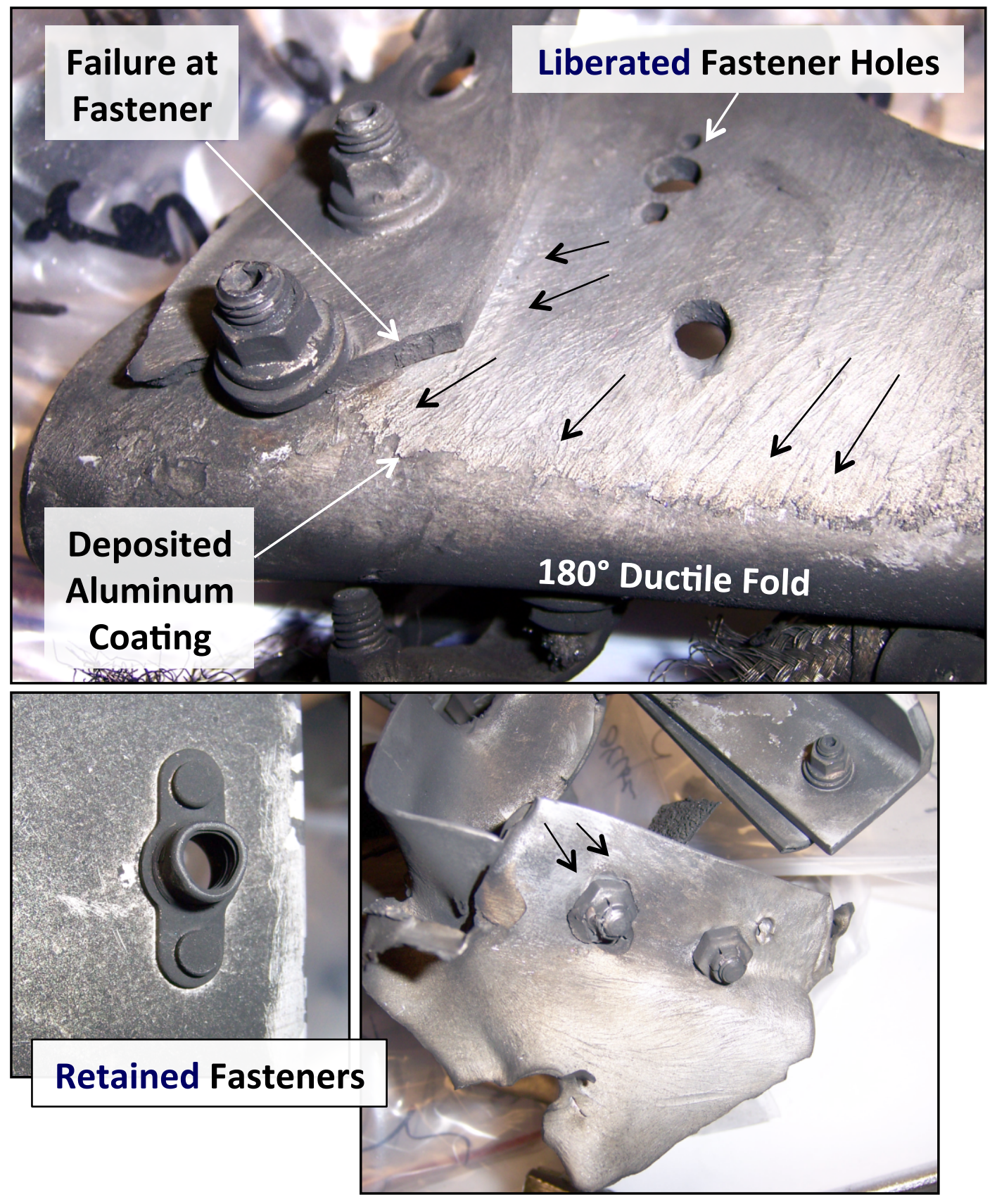

- Very few impact craters were found on the Nutation Control Thruster (NCT)

- Suggests that the majority of the outer cone of primary debris was intercepted by the (interior of) the DebrisLV main tank

- Observed both ductile and brittle failure modes, consistent with many different materials of DebrisLV

- No "missing" small fragments at brittle-failure sites; implies low probability of non-PFC small fragment yields for metallic structures.

- Aluminum liquid condensate flow directions shown by arrows

${ }_{38}$ Liquid-phase path to small fragments (untrackable on orbit) 


\section{Metal Vapor: Upper Stage Titanium Tank Reentry}

- Prior observation of liquid-metal blow-off mechanism generating small, untrackable particles in space.

- High-energy, vacuum conditions

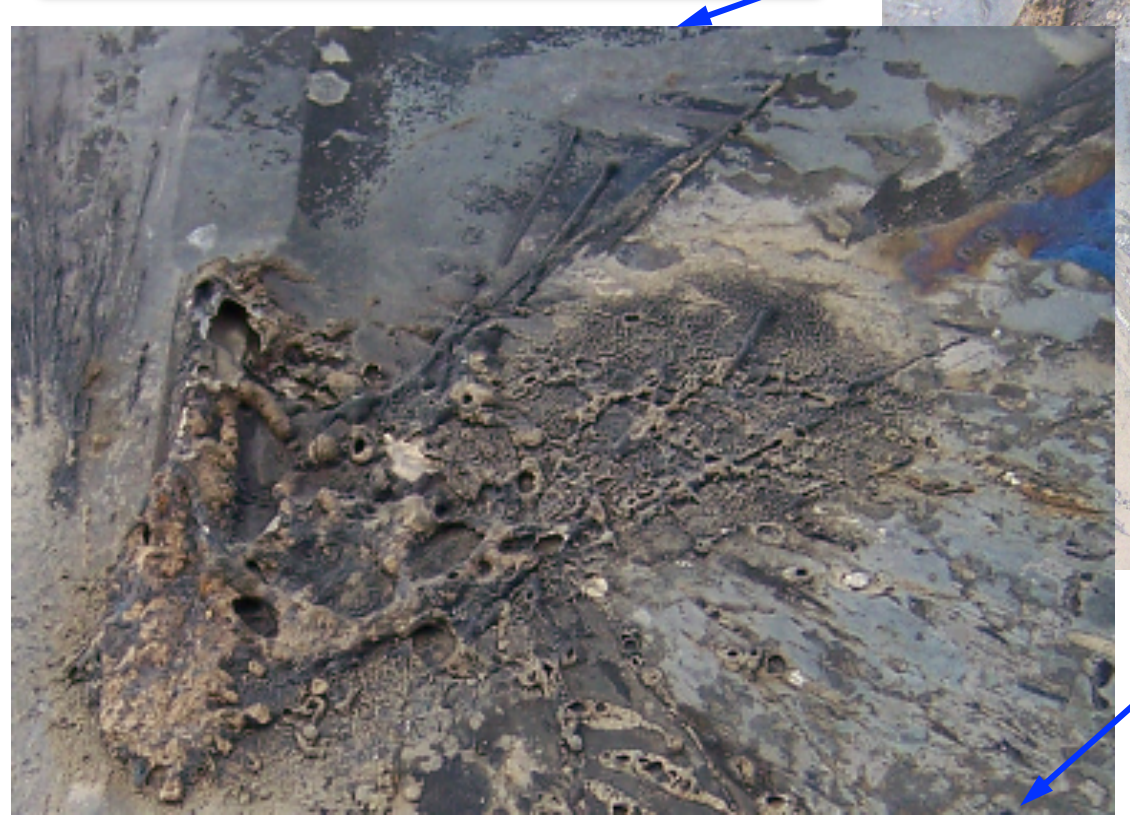

- However; unlike orbital collisions, reentry is below orbital altitudes -metal blow-off particles created by orbital impacts at orbital altitudes would tend to persist in orbit as orbital debris.

Liquid-phase path to small, untrackable fragments

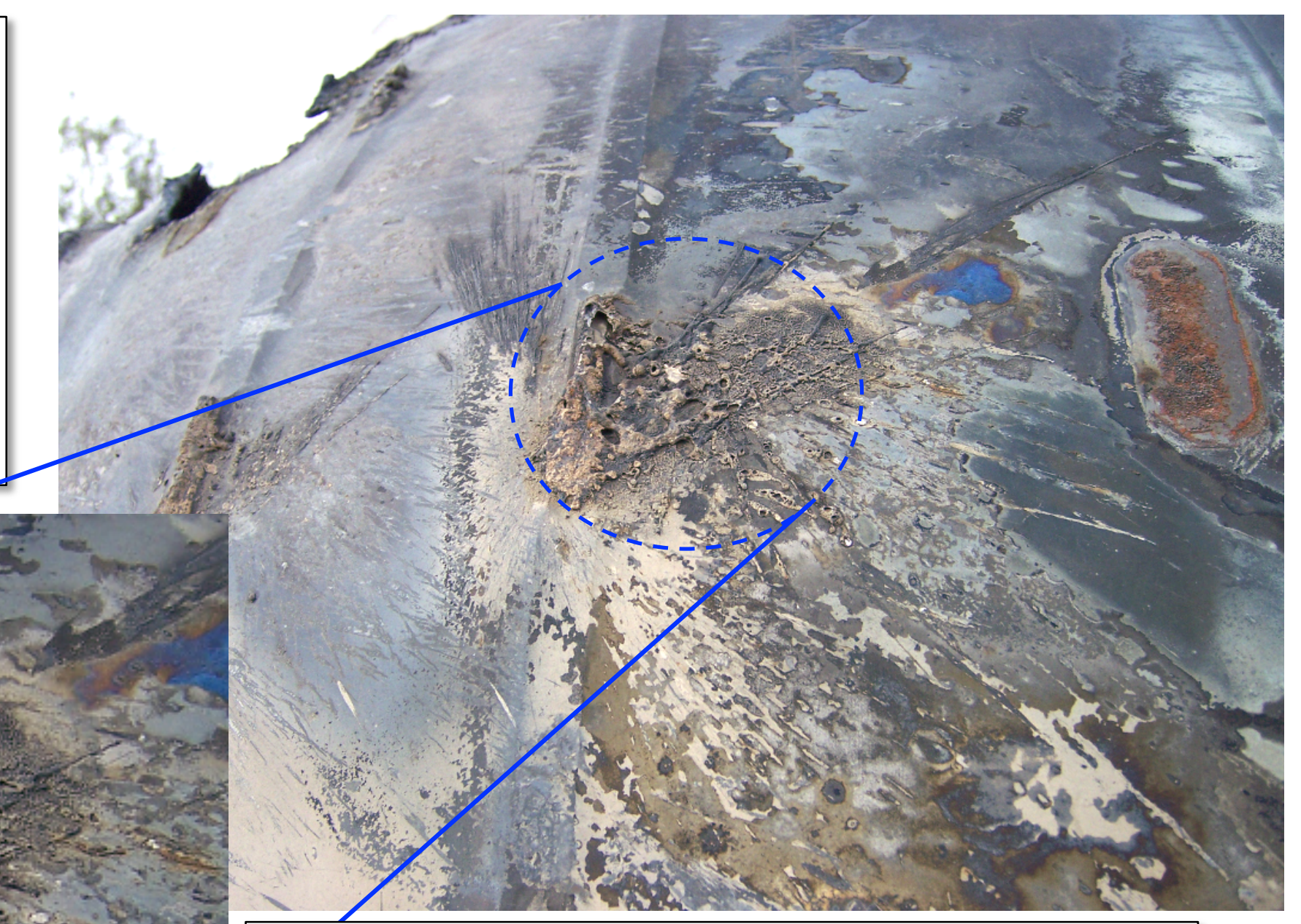




\section{Small, Untrackable Particles In Orbit - Lethality Effect}
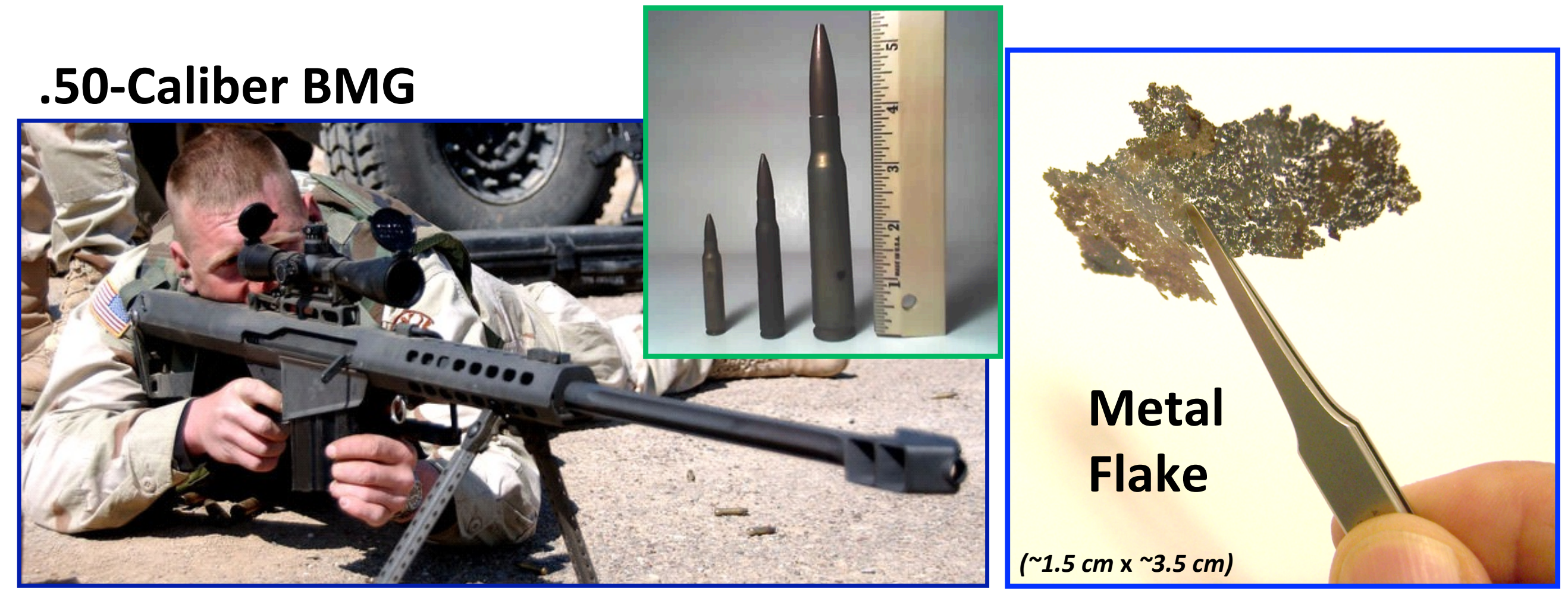

\begin{tabular}{|c|c|c|c|c|}
\hline & $\frac{\text { mass }}{k g}$ & $\frac{\text { velocity }}{\mathrm{m} / \mathrm{s}}$ & $\frac{\text { Energy }}{1 / 2 m v^{2}}$ & $\frac{\text { Momentum }}{m v}$ \\
\hline .357 magnum (HL) & 0.01 & 453 & 1,026 Joules & 4.5 Newton-sec \\
\hline .50 caliber BMG & 0.045 & 910 & 18,632 Joules & 41 Newton-sec \\
\hline Space Flake & 0.00025 & 14000 & 24,500 Joules & 4 Newton-sec \\
\hline
\end{tabular}

- Momentum is always conserved

- Energy may change form into light, sensible-heat, latent-heat, etc.)

Small particles can impact external fuel tanks to initiate orbital explosions. 


\section{MATERIALS PROPERTIES EFFECTS ON FRAGMENTATION}

- Materials strength properties correlate with debris size distribution

- Measure fraction of remaining mass in largest fragment of:

- Aluminum main tank

- Aluminum strap-on tanks

- Copper longeron

- Titanium NCT thruster tank 


\section{Reminder: DebrisLV Materials}

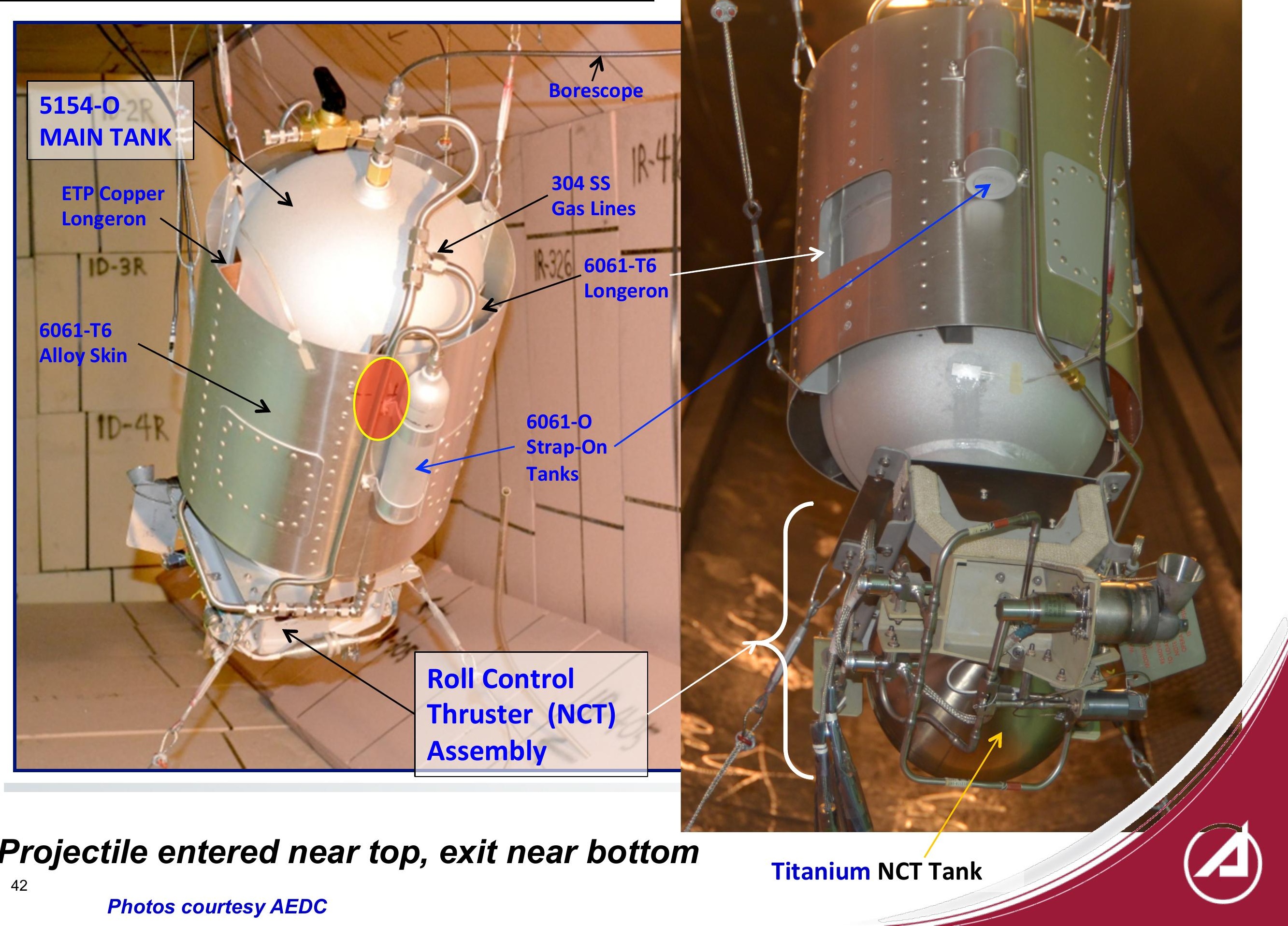




\section{Observed Mass Fraction vs. Structure/Properties}

- Correlation of fragmentation yield with materials physics

- Effects of structure and geometry

$>$ Type of fastener

$>$ Material thickness

$>$ Layout (trusses, construction stresses, etc.)

- Effects of materials properties

$>$ Yield and ultimate strengths, fracture toughness, etc.

$>$ Physical properties (thermal conductivity, melt/boil temperatures, latent heats, etc.)

- As an initial observation, we define "substructures" of DebrisLV, then correlate the substructure mass fraction contained in the largest piece from each substructure

$>$ Longerons $=$ ETP copper, 304 stainless steel, 6061-T6; Main Tank = 5154-0; NCT Tank = 6Al-4V titanium; Strap-on Tanks $=6061-T 6$

$>$ Substructure mass fraction contained in largest fragment from a substructure should vary approximately inversely with the number of small fragments formed by hypervelocity impact (e.g., power law scaling) 


\section{Substructure Yield for Four DebrisLV Substructures}

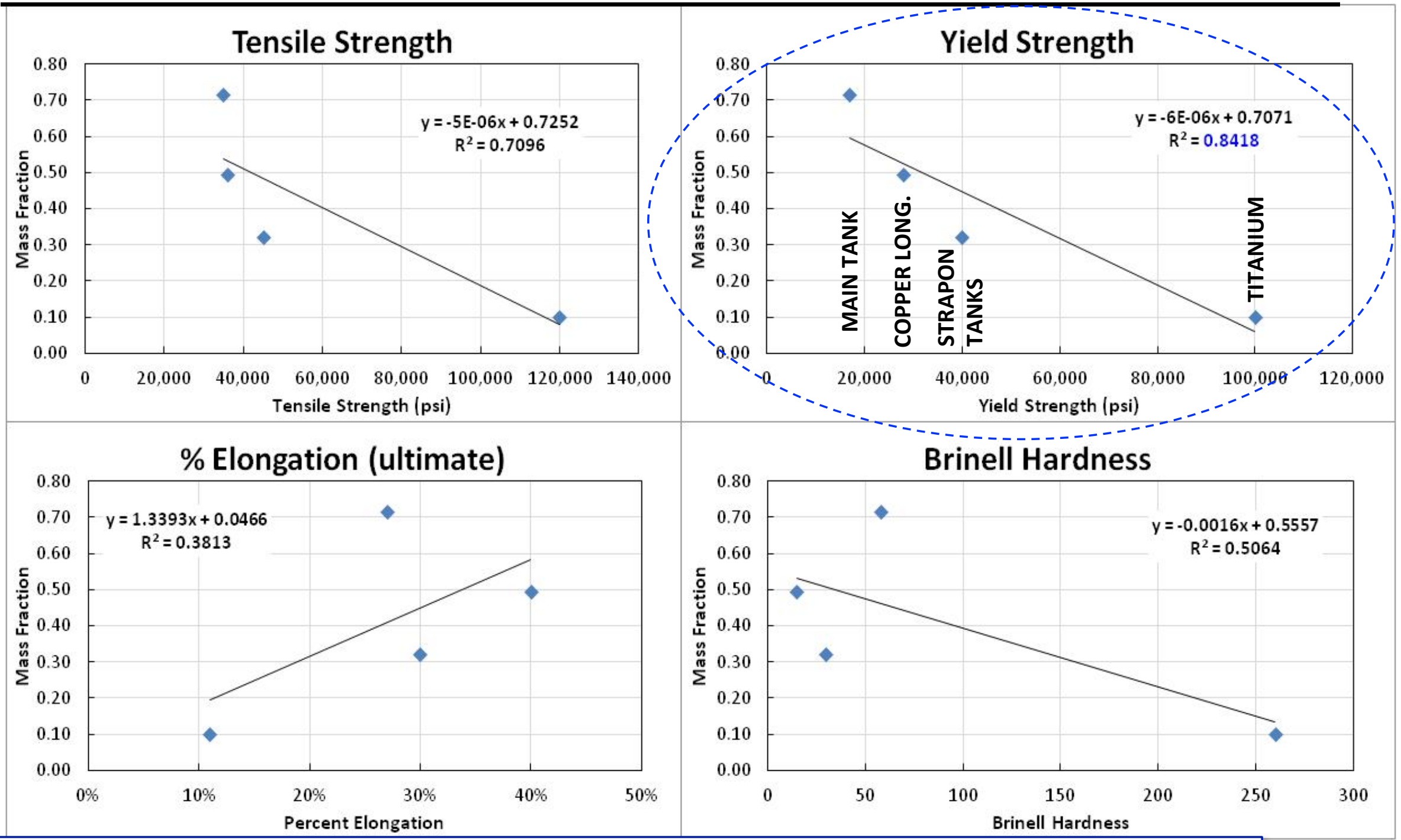

- Substructure mass fraction of the largest piece from 4 different substructures, as a function of literature values of material properties.

- Yield Strength correlates best with "resistance to fragmentation"

- Needs More/Better Data! (e.g., X-Ray data from foam panels that may be available in the future from Univ. of Florida/NASA) 


\section{TECHNICAL CONCLUSIONS}

- The Primary Fragment Cone (PFC) is observed to subsume a zone of relatively complete destruction, due in large part to localized, hypervelocity, condensed-phase bolide (primary fragment) impacts. The resulting cratering patterns are diagnostic and significant mass is missing.

- The physics of condensed-phase impacts is relatively well-reproduced with hydrocodes, as are condensed-phase Jets.

- Fast, high-energy vapor/plasma clouds from Jets propagate at unusual angles from impacts, cause significant structural damage, and launch fragments into unanticipated orbits. These jets are an unanticipated, primary fragmentation, impulse-transfer mechanism in complex structures.

- Liquid metal condensed from the plasma/vapor phase forms large quantities of untrackable fragments, many of which contain sufficient energy to pierce fuel tanks of orbiting structures and initiate explosions.

- Rivets are not preferentially liberated (needs further verification)

- Metal yield strength was observed to correlate moderately well with largest fragment size in specific DebrisLV substructures

$>\quad$ More data is needed

$>$ Only applies to fragments produced outside of the Primary Fragment Cone 


\section{FUNCTIONAL CONCLUSIONS}

- The Aerospace Space Sciences Applications Laboratory has developed a comprehensive, Full-Scale Hypervelocity Impact Experiment test and analysis methodology, capable of significantly extending our understanding of orbital impact physics and mechanics. The Aerospace laboratory analysis and forensic techniques yield useful, specific results which can be incorporated into models to enhance orbital impact fragmentation model accuracy and comprehensiveness.

- We have demonstrated a method to leverage Hydrocode model results to predict plasm jet formation in relatively simple structures. More modeling needs to be done to fully leverage Hydrocode results on the DebrisLV vehicle impact test results.

Plasma jets play an important role in fragmentation energy-transfer during orbital collisions

$>$ Improved knowledge of plasma Jet formation may aid orbital impact analyses

$>$ Although not yet applicable to complex structures, current Hydrocode results help interpret, encapsulate, and reinforce the Debris $L V$ forensic observations and analyses.

- The entire Orbital Debris community needs to be apprised of these results via publication in the open scientific literature. 


\section{RECOMMENDATIONS}

- Obtain panel X-ray information from University of Florida to complete fragment size analysis of DebrisLV

$>$ Liberation of individual rivets; implied nature of liberating force(s)

$>$ Nature of fragments contained in soft-catch

- Present/publish observations to the wider community, SMC, refereed journal articles, and international space debris community

- Repeat full-scale experiment(s) as Targets of Opportunity at AEDC permit: (anticipate 35; current list)

$>$ Study material yield strength dependency (i.e., high-strength main tank)

$>$ Heterogeneous projectile experiment (e.g., steel/titanium/tungsten) is essential to assess partitioning of energy between projectile and target

$>$ Tank containing no gas (DebrisLV contained 15 psia xenon)

$>$ Additional experiments TBD in consultation with DebriSat/DebrisLV Team

- Collaborate with other experimenters

- Current hydrocodes reproduce the Primary Fragment Cone reasonably well, but not observed plasma/vapor blasts

$>$ In-house hydrocode modifications may be fruitful

$>$ Collaborate with other hydrocoders to validly predict plasma jet formation and distinguishing phenomena 


\section{REFERENCES}

1. M. J. Listner, Inter-Agency Space Debris Coordination Committee, http://www.spacesafetymagazine.com/ 2013/02/14/report-space-debris-low-earth-orbit-reaching-tipping-point/

2. Sorge and Mains, "IMPACT Fragmentation Model Improvements," AIAA/AAS Astrodynamics Specialist Conf., San Diego, CA (2014)

3. NASA/TP-2014-217370, “NASA Orbital Debris Engineering Model ORDEM 3.0 - User's Guide," (2014)

4. K. Ruhl, K.D. Bunte, A. Gaede, A. Miller, “ESABASE2 - Debris Software User Manual," (2013)

5. P. Adams, et.al., FTIR Analyses of Hypervelocity Impact Deposits: DebriSat Tests, TOR-2015-00941 (2015), The Aerospace Corporation, El Segundo, CA.

6. P. Adams, et.al., Debris-LV Laboratory Analyses, TOR-2015-00928 (2015), The Aerospace Corporation, El Segundo, CA.

7. N. Hemmi, DebriSat Hypervelocity Impact Fragmentation Modeling, ATM-2014-03659 (2014), The Aerospace Corporation, El Segundo, CA.

8. M. Sorge, "Satellite Fragmentation Modeling with IMPACT," AIAA Astrodyn. Conf., Honolulu (2008)

9. B. Jean and T. Rollins, "Radiation from Hypervelocity Impact Generated Plasma," AIAA Journal, 8(10) (1970).

10. S. Sugita and P. Schultz, "Spectroscopic characterization of hypervelocity jetting: Comparison with a standard theory," J. Geophys. Res., 104( El2) (1999).

11. G. Radhakrishnan, Time-resolved Spectroscopy of Hypervelocity Impact Flash on DebriSat, TOR-2014-03021 (2014), The Aerospace Corporation, El Segundo, CA.

12. P. Adams, et.al., "DebriSat Pre Preshot Laboratory Analyses," TOR-2014-03083 (2014)

13. P. Sheaffer, et.al., "DebrisLV Hypervelocity Impact Experiment Design and Post-Shot Materials Physics Results," TOR-2014-03192 


\section{DebrisLV Hypervelocity Impact Post-Shot Physical Results Summary}

Approved Electronically by:

Anthony T. Salvaggio, PRINC DIRECTOR

ENGINEERING DIRECTORATE

ENGINEERING \& INTEGRATION DIVISION

David J. Gorney, EXECUTIVE VP

OFFICE OF EVP/SSG

(C) The Aerospace Corporation, 2015.

All trademarks, service marks, and trade names are the property of their respective owners. 


\section{External Distribution}

REPORT TITLE

DebrisLV Hypervelocity Impact Post-Shot Physical Results Summary

\begin{tabular}{|c|c|c|}
\hline TOR-2014-03577 & $\begin{array}{l}\text { PUBLICATION DATE } \\
\text { February } 27,2015\end{array}$ & $\begin{array}{l}\text { SECURITY CLASSIFICATION } \\
\text { UNCLASSIFIED }\end{array}$ \\
\hline
\end{tabular}

Thomas Huynh

$\mathrm{SMC} / \mathrm{ENC}$

Thomas.Huynh@losangeles.af.mil

Jesse Edward

$\mathrm{SMC} / \mathrm{ENC}$

jesse.edwards.4@us.af.mil

DATE 